\title{
On generalized fractional integral inequalities for the monotone weighted Chebyshev functionals
}

\author{
Gauhar Rahman ${ }^{1}$, Kottakkaran Sooppy Nisar ${ }^{2}$, Behzad Ghanbari ${ }^{3,4}$ and Thabet Abdeljawad ${ }^{5,6,7^{*}}$ (D)
}

"Correspondence:

tabdeljawad@psu.edu.sa

${ }^{5}$ Department of Mathematics and

General Sciences, Prince Sultan University, P.O. Box 66833, Riyadh,

11586, Kingdom of Saudi Arabia

${ }^{6}$ Department of Medical Research,

China Medical University, Taichung

40402, Taiwan

Full list of author information is

available at the end of the article

\begin{abstract}
In this paper, we establish the generalized Riemann-Liouville (RL) fractional integrals in the sense of another increasing, positive, monotone, and measurable function $\Psi$. We determine certain new double-weighted type fractional integral inequalities by utilizing the said integrals. We also give some of the new particular inequalities of the main result. Note that we can form various types of new inequalities of fractional integrals by employing conditions on the function $\Psi$ given in the paper. We present some corollaries as particular cases of the main results.
\end{abstract}

Keywords: Fractional integrals; The generalized fractional integrals; Fractional integral inequalities; The Chebyshev functional

\section{Introduction}

The Chebyshev functional is given by $($ see $[7,10])$

$$
\begin{aligned}
\mathscr{T}(\mathscr{U}, \mathscr{V}, \mu)= & \int_{x_{1}}^{x_{2}} \mu(\tau) d \tau \int_{x_{1}}^{x_{2}} \mu(\tau) \mathscr{U}(\tau) \mathscr{V}(\tau) d \tau \\
& -\int_{x_{1}}^{x_{2}} \mu(\tau) \mathscr{U}(\tau) d \tau \int_{x_{1}}^{x_{2}} \mu(\tau) \mathscr{V}(\tau) d \tau,
\end{aligned}
$$

where $\mathscr{U}$ and $\mathscr{V}$ are integrable functions on $\left[x_{1}, x_{2}\right]$, and $\mu$ is a positive integrable function on $\left[x_{1}, x_{2}\right]$. Applications of functional (1) are found in probability and statistical problems. Further applications can be found in $[6,16,36]$. In $[9,35]$ the authors defined the following extended Chebyshev functional:

$$
\begin{aligned}
\mathscr{T}(\mathscr{U}, \mathscr{V}, \mu, \nu)= & \int_{x_{1}}^{x_{2}} \nu(\tau) d \tau \int_{x_{1}}^{x_{2}} \mu(\tau) \mathscr{U}(\tau) \mathscr{V}(\tau) d \tau \\
& +\int_{x_{1}}^{x_{2}} \mu(\tau) d \tau \int_{x_{1}}^{x_{2}} \nu(\tau) \mathscr{U}(\tau) \mathscr{V}(\tau) d \tau
\end{aligned}
$$

(c) The Author(s) 2020. This article is licensed under a Creative Commons Attribution 4.0 International License, which permits use, sharing, adaptation, distribution and reproduction in any medium or format, as long as you give appropriate credit to the original author(s) and the source, provide a link to the Creative Commons licence, and indicate if changes were made. The images or other third party material in this article are included in the article's Creative Commons licence, unless indicated otherwise in a credit line to the material. If material is not included in the article's Creative Commons licence and your intended use is not permitted by statutory regulation or exceeds the permitted use, you will need to obtain permission directly from the copyright holder. To view a copy of this licence, visit http://creativecommons.org/licenses/by/4.0/. 


$$
\begin{aligned}
& -\int_{x_{1}}^{x_{2}} \mu(\tau) \mathscr{U}(\tau) d \tau \int_{x_{1}}^{x_{2}} \nu(\tau) \mathscr{V}(\tau) d \tau \\
& -\int_{x_{1}}^{x_{2}} \nu(\tau) \mathscr{U}(\tau) d \tau \int_{x_{1}}^{x_{2}} \mu(\tau) \mathscr{V}(\tau) d \tau,
\end{aligned}
$$

where $\mathscr{U}$ and $\mathscr{V}$ are integrable functions on $\left[x_{1}, x_{2}\right]$, and $\mu$ and $v$ are positive integrable functions on $\left[x_{1}, x_{2}\right]$. The functions $\mathscr{U}$ and $\mathscr{V}$ are said to be synchronous on $\left[x_{1}, x_{2}\right]$ if

$$
(\mathscr{U}(\rho)-\mathscr{U}(\zeta))(\mathscr{V}(\rho)-\mathscr{V}(\zeta)) \geq 0, \quad \rho, \zeta \in\left[x_{1}, x_{2}\right]
$$

The functions $\mathscr{U}$ and $\mathscr{V}$ are said to be asynchronous on $\left[x_{1}, x_{2}\right]$ if the inequality reversed, that is,

$$
(\mathscr{U}(\rho)-\mathscr{U}(\zeta))(\mathscr{V}(\rho)-\mathscr{V}(\zeta)) \leq 0, \quad \rho, \zeta \in\left[x_{1}, x_{2}\right]
$$

If the functions $\mathscr{U}$ and $\mathscr{V}$ are synchronous on $[r, s]$, then $\mathscr{T}(\mathscr{U}, \mathscr{V}, \mu) \geq 0$ and $\mathscr{T}(\mathscr{U}, \mathscr{V}$, $\mu, v) \geq 0$. For further details, the reader may consult Kuang [27] and Mitrinovic [35]. If we consider $\mu(\vartheta)=v(\vartheta)=1, \vartheta \in\left[x_{1}, x_{2}\right]$, then $\mathscr{T}(\mathscr{U}, \mathscr{V}, \mu)=\frac{1}{2} \mathscr{T}(\mathscr{U}, \mathscr{V}, \mu, v)$. In $[3,12,34,44]$, various researchers gave valuable consideration to functionals (1) and (2). Recently, Rahman et al. [57] defined fractional conformable inequalities for the Chebyshev functionals (1) and (2).

Awan et al. [2] presented the following result: If $\Phi$ is an absolutely continuous on $\left[x_{1}, x_{2}\right]$ such that $\left(\Phi^{\prime}\right)^{2} \in L_{1}\left[x_{1}, x_{2}\right]$ and $\mu$ is a positive integrable function on $\left[x_{1}, x_{2}\right]$, then the following inequality holds;

$$
\begin{aligned}
\mathscr{T}(\Phi, \Phi, \mu) \leq & \frac{1}{Q^{2}\left(x_{2}\right)} \int_{x_{1}}^{x_{2}}\left[\int_{x_{1}}^{\tau} \mu(\tau) d \tau \int_{x_{1}}^{x_{2}} \tau \mu(\tau) d \tau-\int_{x_{1}}^{x_{2}} \mu(\tau) d \tau \int_{x_{1}}^{\tau} \tau \mu(\tau) d \tau\right] \\
& \times\left[\Phi^{\prime}(\theta)\right]^{2} d \theta
\end{aligned}
$$

where $Q\left(x_{2}\right)=\int_{x_{1}}^{x_{2}} \mu(\tau) d \tau$.

Bezziou et al. [5] presented the following result.

Theorem 1.1 Let $\Phi:\left[x_{1}, x_{2}\right] \rightarrow \mathbb{R}$ be an absolutely continuous function such that $\left(\Phi^{\prime}\right)^{2} \in$ $L_{1}\left[x_{1}, x_{2}\right]$, and let $\mu:\left[x_{1}, x_{2}\right] \rightarrow \mathbb{R}^{+}$be an integrable function. Then we have the following inequality for $\kappa>0$ :

$$
\mathscr{J}_{x_{1}}^{\tau} \mu\left(x_{2}\right) \mathscr{J}_{x_{1}}^{\tau} \mu \Phi^{2}\left(x_{2}\right)-\left(\mathscr{J}_{x_{1}}^{\tau} \mu \Phi\left(x_{2}\right)\right)^{2} \leq \int_{x_{1}}^{x_{2}} \Lambda(\theta)\left[\Phi^{\prime}(\theta)\right]^{2} d \theta
$$

with

$$
\Lambda(\theta)=\frac{1}{2}\left[\mathscr{J}_{x_{1}}^{\tau} x_{2} \mu\left(x_{2}\right) \int_{x_{1}}^{\tau}\left(x_{2}-\tau\right)^{\tau-1} \mu(\tau) d \tau-\mathscr{J}_{x_{1}}^{\tau} \mu\left(x_{2}\right) \int_{x_{1}}^{\tau} \tau\left(x_{2}-\tau\right)^{\tau-1} \mu(\tau) d \tau\right],
$$

where $\mathscr{J}_{x_{1}}^{\tau}$ is the classical RL-fractional integral.

Dahmani and Bounoua [13] established the following result. 
Theorem 1.2 Let $\Phi:\left[x_{1}, x_{2}\right] \rightarrow \mathbb{R}$ be an absolutely continuous such that $\left(\Phi^{\prime}\right)^{2} \in L_{1}\left[x_{1}, x_{2}\right]$, and let $\mu:\left[x_{1}, x_{2}\right] \rightarrow \mathbb{R}^{+}$be an integrable function. Then for all $\kappa>0$ and $\theta \in\left[x_{1}, x_{2}\right]$, the following inequality holds;

$$
\begin{aligned}
& \frac{1}{\mathscr{J}_{x_{1}}^{\tau} \mu(\theta)} \mathscr{J}_{x_{1}}^{\tau}\left(\mu \Phi^{2}\right)(\theta)-\left[\frac{1}{\mathscr{J}_{x_{1}}^{\tau} \mu(\theta)} \mathscr{J}_{x_{1}}^{\tau}(\mu \Phi)(\theta)\right]^{2} \\
& \leq \frac{1}{\left[\mathscr{J}_{x_{1}}^{\tau} \mu(\theta)\right]^{2}} \int_{x_{1}}^{\theta} Q_{\theta}(\tau)\left[\Phi^{\prime}(\tau)\right]^{2} d \tau
\end{aligned}
$$

with

$$
\begin{aligned}
Q_{\theta}(\tau)= & \frac{1}{\Gamma(\tau)}\left[\mathscr{J}_{x_{1}}^{\tau}(\theta \mu(\theta)) \int_{x_{1}}^{\rho} \mu(\vartheta)(\theta-\vartheta)^{\tau-1} d \vartheta\right. \\
& \left.-\mathscr{J}_{x_{1}}^{\kappa} \mu(\theta) \int_{x_{1}}^{\rho} \vartheta \mu(\vartheta)(\theta-\vartheta)^{\tau-1} d \vartheta\right],
\end{aligned}
$$

where $\mathscr{J}_{x_{1}}^{\tau}$ is the classical Riemann-Liouville fractional integral.

In the last few decades, the researchers investigated different kinds of integral inequalities by considering various integral approaches. In [14] the authors gave weighted Grüsstype inequalities by taking RL-fractional integrals into account. Dahmani [8] proposed some new inequalities in the sense of fractional integrals. Several inequalities for the extended gamma function and confluent hypergeometric $k$-function are found by Nisar et al. [38]. Nisar et al. [39] used Riemann-Liouville and Hadamard $k$-fractional derivatives and investigated Gronwall-type inequalities with applications. Rahman et al. [55] studied $(k, \rho)$-fractional integrals and investigated the corresponding inequalities. Sarikaya and Budak [59] proposed Ostrowski-type inequalities by considering local fractional integrals. Sarikaya et al. [60] proposed the idea of generalized $(k, s)$-fractional integrals with applications. Set et al. [61] investigated Grüss-type inequalities for the generalized $k$-fractional integrals. Recently, Jarad et al. [22, 23] proposed the idea of fractional conformable and proportional fractional integral operators. Huang et al. [20] recently presented generalized Hermite-Hadamard-type inequalities for $k$-fractional conformable integrals. Qi et al. [45] proposed Chebyshev-type inequalities by using generalized $k$-fractional conformable integrals. Rahman et al. [56] investigated Chebyshev-type inequalities by utilizing fractional conformable integrals. Chebyshev-type inequalities and Minkowski-type inequalities involving generalized conformable integrals can be found in the work of Nisar et al. [42, 43]. Recently, Tassaddiq et al. [63] proposed certain inequalities for the weighted and extended Chebyshev functionals by using fractional conformable integrals. Nisar et al. [40] presented some new classes of inequalities for an $n(n \in \mathbb{N})$ family of positive continuous and decreasing functions via generalized conformable fractional integrals. Nisar et al. [41] established generalized fractional integral inequalities via the Marichev-Saigo-Maeda (MSM) fractional integral operators. Rahman et al. [54] recently investigated Grüss-type inequalities for generalized $k$-fractional conformable integrals. Minkowski's inequalities, fractional Hadamard proportional integral inequalities, and fractional proportional inequalities for convex functions by employing fractional proportional integrals can be found in [46-53]. In addition, various applications of fractional calculus can be found in $[1,17,18,28-32,62,64]$. 
The paper is organized as follows. Some auxiliary results are presented in Sect. 2. In Sect. 3, we present double-weighted fractional integral inequalities for the Chebyshev functionals. In Sect. 4, we retrieve several particular cases of the results. A concluding remark is given in Sect. 5 .

\section{Auxiliary results}

In this section, we present some well-known definitions and mathematical preliminaries of fractional calculus.

Definition 2.1 ([26, 58]) Let $\mathscr{U} \in L\left[x_{1}, x_{2}\right]$. Then the classical left- and right-sided RLfractional integrals of order $\tau>0$ and $x_{1} \geq 0$ are respectively defined by

$$
\left(x_{1} \mathscr{J}^{\tau} \mathscr{U}\right)(\vartheta)=\frac{1}{\Gamma(\tau)} \int_{a}^{\vartheta}(\vartheta-\varrho)^{\tau-1} \mathscr{U}(\varrho) d \varrho, \quad x_{1}<\vartheta
$$

and

$$
\left(\mathscr{J}_{x_{2}}^{\tau} \mathscr{U}\right)(\vartheta)=\frac{1}{\Gamma(\tau)} \int_{\vartheta}^{x_{2}}(\varrho-\vartheta)^{\tau-1} \mathscr{U}(\varrho) d \varrho, \quad \vartheta<x_{2},
$$

where $\Gamma$ is the standard gamma function.

Definition 2.2 ([37]) Let $\mathscr{U} \in L\left[x_{1}, x_{2}\right]$. Then the generalized left- and right-sided RL $\kappa$ fractional integrals of order $\tau>0$ and $x_{1} \geq 0$ are respectively defined by

$$
\left(x_{1} \mathscr{J}_{\kappa}^{\tau} \mathscr{U}\right)(\vartheta)=\frac{1}{\kappa \Gamma_{\kappa}(\tau)} \int_{a}^{\vartheta}(\vartheta-\varrho)^{\frac{\tau}{\kappa}-1} \mathscr{U}(\varrho) d \varrho, \quad x_{1}<\vartheta
$$

and

$$
\left(\mathscr{J}_{x_{2}, \kappa}^{\tau} \mathscr{U}\right)(\vartheta)=\frac{1}{\kappa \Gamma_{\kappa}(\tau)} \int_{\vartheta}^{x_{2}}(\varrho-\vartheta)^{\frac{\tau}{\kappa}-1} \mathscr{U}(\varrho) d \varrho, \quad \vartheta<x_{2},
$$

where $\Gamma_{\kappa}$ is the $\kappa$-gamma function defined in [15].

Remark 2.1 Applying Definition 2.2 for $\kappa=1$, we get Definition 2.1.

Definition 2.3 ([26]) Let $\mathscr{U}:\left[x_{1}, x_{2}\right] \rightarrow \mathbb{R}$ be an integrable function, and let $\Psi$ be an increasing positive function on $\left(x_{1}, x_{2}\right]$ with continuous derivative $\Psi^{\prime}$ on $\left(x_{1}, x_{2}\right)$. Then the left- and right-sided generalized RL fractional integrals of a function $\mathscr{U}$ concerning another function $\Psi$ are respectively defined by

$$
\left(\begin{array}{l}
\Psi \\
x_{1}
\end{array} \mathscr{J}^{\tau} \mathscr{U}\right)(\rho)=\frac{1}{\Gamma(\tau)} \int_{x_{1}}^{\vartheta}(\Psi(\vartheta)-\Psi(\varrho))^{\frac{\tau}{\kappa}-1} \Psi^{\prime}(\varrho) \mathscr{U}(\varrho) d \varrho, \quad x_{1}<\vartheta
$$

and

$$
\left({ }^{\Psi} \mathscr{J}_{x_{2}}^{\tau} \mathscr{U}\right)(\rho)=\frac{1}{\Gamma(\tau)} \int_{\vartheta}^{x_{2}}(\Psi(\varrho)-\Psi(\vartheta))^{\frac{\tau}{\kappa}-1} \Psi^{\prime}(\varrho) \mathscr{U}(\varrho) d \varrho, \quad \vartheta<x_{2},
$$

where $\kappa>0$ and $\tau \in \mathbb{C}$ with $\Re(\tau)>0$. 
Definition 2.4 ([33]) Let $\mathscr{U}:\left[x_{1}, x_{2}\right] \rightarrow \mathbb{R}$ be an integrable function, and let $\Psi$ be an increasing positive function on $\left(x_{1}, x_{2}\right]$ with continuous derivative $\Psi^{\prime}$ on $\left(x_{1}, x_{2}\right)$. Then the left- and right-sided generalized RL $\kappa$-fractional integrals of a function $\mathscr{U}$ concerning another function $\Psi$ are respectively defined by

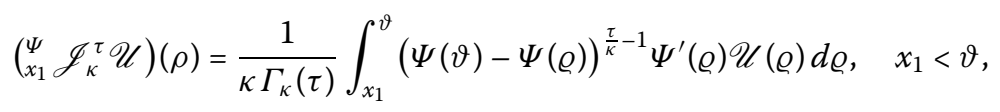

and

$$
\left({ }^{\Psi} \mathscr{J}_{x_{2}, \kappa}^{\tau} \mathscr{U}\right)(\rho)=\frac{1}{\kappa \Gamma_{\kappa}(\tau)} \int_{\vartheta}^{x_{2}}(\Psi(\varrho)-\Psi(\vartheta))^{\frac{\tau}{\kappa}-1} \Psi^{\prime}(\varrho) \mathscr{U}(\varrho) d \varrho, \quad \vartheta<x_{2},
$$

where $\kappa>0$ and $\tau \in \mathbb{C}$ with $\Re(\tau)>0$.

Remark 2.2 The following particular cases are easily derived:

i. Applying Definition 2.4 for $\Psi(\vartheta)=\vartheta$, we get Definition 2.2,

ii. Applying Definition 2.4 for $\kappa=1$, we get Definition 2.3,

iii. Applying Definition 2.4 for $\Psi(\vartheta)=\ln \vartheta$, we get the generalized Hadamard $\kappa$-fractional integrals defined in [21],

iv. Applying Definition 2.4 for $\Psi(\vartheta)=\ln \vartheta$ and $\kappa=1$ leads to the Hadamard fractional integrals defined in [26],

v. Applying Definition 2.4 for $\Psi(\vartheta)=\frac{\vartheta^{\tau}}{\tau}, \tau>0$, and $\kappa=1$ leads to the Katugampola fractional integrals [24],

vi. Applying Definition 2.4 for $\Psi(\vartheta)=\frac{\vartheta^{\alpha+s}}{\alpha+s}$ and $\kappa=1$ (where $\alpha \in(0,1], s \in \mathbb{R}$, and $\mu+s \neq 0$ ) leads to the generalized fractional conformable integrals defined by Khan and Khan [25],

vii. Applying Definition 2.4 for $\Psi(\vartheta)=\frac{\left(\vartheta-x_{1}\right)^{\alpha}}{\alpha}$ and $\Psi(\vartheta)=\frac{-\left(x_{2}-\vartheta\right)^{\alpha}}{\alpha}, \alpha>0$, leads to the $(k, \alpha)$-fractional conformable integrals defined by Habib et al. [19].

viii. Applying Definition 2.4 for $\Psi(\vartheta)=\frac{\left(\vartheta-x_{1}\right)^{\alpha}}{\alpha}, \Psi(\vartheta)=\frac{-\left(x_{2}-\vartheta\right)^{\alpha}}{\alpha}, \alpha>0$, and $\kappa=1$ leads to the conformable fractional integrals defined by Jarad et al. [23],

ix. Applying Definition 2.4 for $\Psi(\vartheta)=\vartheta$ and $\kappa=1$, we get Definition 2.1.

\section{Some double-weighted generalized fractional integral inequalities}

In this section, we present some double-weighted generalized fractional integral inequalities. We start by proving the following lemma.

Lemma 3.1 Let $\Psi$ be a measurable increasing positive function on $\left(x_{1}, x_{2}\right)$ with continuous derivative $\Psi^{\prime}(\varrho)$ on $\left[x_{1}, x_{2}\right]$. Let $\mathscr{V}:\left[x_{1}, x_{2}\right] \rightarrow \mathbb{R}$ be continuous on $\left[x_{1}, x_{2}\right]$, and let $\mu, v:$ $\left[x_{1}, x_{2}\right] \rightarrow \mathbb{R}^{+}$be positive integrable. Then for all $\tau, \kappa>0$, we have

$$
\begin{aligned}
& {\left[{ }_{x_{1}}^{\Psi}{ }_{\mathbb{I}_{\kappa}}^{\tau} \mu\left(x_{2}\right)\right]\left[{ }_{x_{1}}^{\Psi} \mathscr{I}_{\kappa}^{\tau} x_{2}(v \mathscr{V})\left(x_{2}\right)\right]+\left[{ }_{x_{1}}^{\Psi} \mathscr{I}_{\kappa}^{\tau} \mathscr{V}\left(x_{2}\right)\right]\left[{ }_{x_{1}}^{\Psi} \mathscr{I}_{\kappa}^{\tau} x_{2}(\mu \mathscr{V})\left(x_{2}\right)\right] } \\
& \quad-\left[{ }_{x_{1}}^{\Psi} \mathscr{I}_{\kappa}^{\tau} \mu\left(x_{2}\right) x_{2} \mu\left(x_{2}\right)\right]\left[{ }_{x_{1}}^{\Psi} \mathscr{I}_{\kappa}^{\tau}(v \mathscr{V})\left(x_{2}\right)\right]-\left[{ }_{x_{1}}^{\Psi} \mathscr{I}_{\kappa}^{\tau} x_{2} v\left(x_{2}\right)\right]\left[{ }_{x_{1}}^{\Psi} \mathscr{I}_{\kappa}^{\tau}(\mu \mathscr{V})\left(x_{2}\right)\right] \\
& \leq \frac{1}{\kappa \Gamma_{\kappa}(\tau)} \int_{x_{1}}^{x_{2}}\left[{ }_{x_{1}}^{\Psi} \mathscr{I}_{\kappa}^{\tau} x_{2} \mu\left(x_{2}\right) \int_{x_{1}}^{\vartheta}\left(\Psi\left(x_{2}\right)-\Psi(\varrho)\right)^{\frac{\tau}{\kappa}-1} \Psi^{\prime}(\varrho) v(\varrho) d \varrho\right. \\
&\left.-{ }_{x_{1}}^{\Psi} \mathscr{I}_{\kappa}^{\tau} \mu\left(x_{2}\right) \int_{x_{1}}^{\vartheta} \varrho\left(\Psi\left(x_{2}\right)-\Psi(\varrho)\right)^{\frac{\tau}{\kappa}-1} \Psi^{\prime}(\varrho) v(\varrho) d \varrho\right]\left(\Psi^{\prime}(\vartheta)\right) d \vartheta .
\end{aligned}
$$


Proof Suppose that $\mathscr{U}:\left[x_{1}, x_{2}\right] \rightarrow \mathbb{R}$ is a continuous function on $\left[x_{1}, x_{2}\right]$. Then we get

$$
\begin{aligned}
\left.\left.{ }_{x_{1}}^{\Psi} \mathscr{I}_{\kappa}^{\tau} \mu\left(x_{2}\right)\right]\left[{ }_{x_{1}}^{\Psi} \mathscr{I}_{\kappa}^{\tau}(v \mathscr{U} \mathscr{V})\left(x_{2}\right)\right]+{ }_{x_{1}}^{\Psi} \mathscr{I}_{\kappa}^{\tau} \mathscr{V}\left(x_{2}\right)\right]\left[{ }_{x_{1}}^{\Psi} \mathscr{I}_{\kappa}^{\tau}(\mu \mathscr{U} \mathscr{V})\left(x_{2}\right)\right] \\
\left.\left.\quad-\left[{ }_{x_{1}}^{\Psi} \mathscr{I}_{\kappa}^{\tau}(\mu \mathscr{U})\left(x_{2}\right)\right]\left[{ }_{x_{1}}^{\Psi} \mathscr{I}_{\kappa}^{\tau}(v \mathscr{V})\left(x_{2}\right)\right]-{ }_{x_{1}}^{\Psi} \mathscr{I}_{\kappa}^{\tau}(\nu \mathscr{U})\left(x_{2}\right)\right][]_{x_{1}}^{\Psi} \mathscr{I}_{\kappa}^{\tau}(\mu \mathscr{V})\left(x_{2}\right)\right] \\
=\frac{1}{\kappa^{2} \Gamma_{\kappa}^{2}(\tau)} \int_{x_{1}}^{x_{2}} \int_{x_{1}}^{x_{2}}\left(\Psi\left(x_{2}\right)-\Psi(\xi)\right)^{\frac{\tau}{\kappa}-1}\left(\Psi\left(x_{2}\right)-\Psi(\varrho)\right)^{\frac{\tau}{\kappa}-1} \\
\quad \times \Psi^{\prime}(\xi) \mu(\xi) \Psi^{\prime}(\varrho) v(\varrho)[(\mathscr{U}(\xi)-\mathscr{U}(\varrho))(\mathscr{V}(\xi)-\mathscr{V}(\varrho))] d \xi d \varrho .
\end{aligned}
$$

Consequently, it follows that

$$
\begin{aligned}
{\left.\left[{ }_{x_{1}}^{\Psi}{ }_{x_{\kappa}} \mathscr{I}_{\kappa}^{\tau} \mu\left(x_{2}\right)\right]\left[{ }_{x_{1}}^{\Psi} \mathscr{I}_{\kappa}^{\tau}(v \mathscr{U} \mathscr{V})\left(x_{2}\right)\right]+{ }_{x_{1}}^{\Psi} \mathscr{I}_{\kappa}^{\tau} \mathscr{V}\left(x_{2}\right)\right]\left[{ }_{x_{1}}^{\Psi} \mathscr{I}_{\kappa}^{\tau}(\mu \mathscr{U} \mathscr{V})\left(x_{2}\right)\right] } \\
\left.\quad-\left[{ }_{x_{1}}^{\Psi} \mathscr{I}_{\kappa}^{\tau}(\mu \mathscr{U})\left(x_{2}\right)\right]\left[{ }_{x_{1}}^{\Psi} \mathscr{I}_{\kappa}^{\tau}(\nu \mathscr{V})\left(x_{2}\right)\right]-{ }_{x_{1}}^{\Psi} \mathscr{I}_{\kappa}^{\tau}(v \mathscr{U})\left(x_{2}\right)\right]\left[{ }_{x_{1}}^{\Psi} \mathscr{I}_{\kappa}^{\tau}(\mu \mathscr{V})\left(x_{2}\right)\right] \\
=\frac{1}{\kappa^{2} \Gamma_{\kappa}^{2}(\tau)} \int_{x_{1}}^{x_{2}} \int_{x_{1}}^{x_{2}}\left(\Psi\left(x_{2}\right)-\Psi(\xi)\right)^{\frac{\tau}{\kappa}-1}\left(\Psi\left(x_{2}\right)-\Psi(\varrho)\right)^{\frac{\tau}{\kappa}-1} \\
\quad \times \Psi^{\prime}(\xi) \mu(\xi) \Psi^{\prime}(\varrho) v(\varrho)(\mathscr{U}(\xi)-\mathscr{U}(\varrho))\left(\int_{\varrho}^{\xi} \mathscr{V}^{\prime}(\vartheta) d \vartheta\right) d \xi d \varrho .
\end{aligned}
$$

Utilizing the condition $x_{1} \leq \varrho \leq \vartheta \leq \xi \leq x_{2}$, we conclude that

$$
\begin{aligned}
{\left.\left[{ }_{x_{1}}^{\Psi} \mathscr{I}_{\kappa}^{\tau} \mu\left(x_{2}\right)\right]\left[{ }_{x_{1}}^{\Psi} \mathscr{I}_{\kappa}^{\tau}(v \mathscr{U} \mathscr{V})\left(x_{2}\right)\right]+{ }_{x_{1}}^{\Psi} \mathscr{I}_{\kappa}^{\tau} \mathscr{V}\left(x_{2}\right)\right]\left[{ }_{x_{1}}^{\Psi} \mathscr{I}_{\kappa}^{\tau}(\mu \mathscr{U} \mathscr{V})\left(x_{2}\right)\right] } \\
\left.\quad-\left[{ }_{x_{1}}^{\Psi} \mathscr{I}_{\kappa}^{\tau}(\mu \mathscr{U})\left(x_{2}\right)\right]\left[{ }_{x_{1}}^{\Psi} \mathscr{I}_{\kappa}^{\tau}(\nu \mathscr{V})\left(x_{2}\right)\right]-{ }_{x_{1}}^{\Psi} \mathscr{I}_{\kappa}^{\tau}(\nu \mathscr{U})\left(x_{2}\right)\right]\left[{ }_{x_{1}}^{\Psi} \mathscr{I}_{\kappa}^{\tau}(\mu \mathscr{V})\left(x_{2}\right)\right] \\
=\frac{1}{\kappa^{2} \Gamma_{\kappa}^{2}(\tau)} \int_{x_{1}}^{x_{2}}\left[\int_{x_{1}}^{\vartheta}\left(\Psi\left(x_{2}\right)-\Psi(\varrho)\right)^{\frac{\tau}{\kappa}-1} \Psi^{\prime}(\varrho) v(\varrho)\right. \\
\left.\quad \times \int_{x_{1}}^{x_{2}}\left(\Psi\left(x_{2}\right)-\Psi(\xi)\right)^{\frac{\tau}{\kappa}-1}(\mathscr{U}(\xi)-\mathscr{U}(\varrho)) \Psi^{\prime}(\xi) \mu(\xi) d \xi d \varrho\right]\left(\mathscr{V}^{\prime}(\vartheta)\right) d \vartheta .
\end{aligned}
$$

Applying (13) to the particular case $\mathscr{U}(x)=x$, we can write

$$
\begin{aligned}
{\left[{ }_{x_{1}}^{\Psi} \mathscr{I}_{\kappa}^{\tau} \mu\left(x_{2}\right)\right]\left[{ }_{x_{1}}^{\Psi} \mathscr{I}_{\kappa}^{\tau} x_{2}(\nu \mathscr{V})\left(x_{2}\right)\right]+\left[\begin{array}{l}
\Psi \\
x_{1}
\end{array} \mathscr{I}_{\kappa}^{\tau} \mathscr{V}\left(x_{2}\right)\right]\left[{ }_{x_{1}}^{\Psi} \mathscr{I}_{\kappa}^{\tau} x_{2}(\mu \mathscr{V})\left(x_{2}\right)\right] } \\
\quad-\left[{ }_{x_{1}}^{\Psi} \mathscr{I}_{\kappa}^{\tau} x_{2}(\mu)\left(x_{2}\right)\right]\left[{ }_{x_{1}}^{\Psi} \mathscr{I}_{\kappa}^{\tau}(\nu \mathscr{V})\left(x_{2}\right)\right]-\left[{ }_{x_{1}}^{\Psi} \mathscr{I}_{\kappa}^{\tau} x_{2}(v)\left(x_{2}\right)\right]\left[{ }_{x_{1}}^{\Psi} \mathscr{I}_{\kappa}^{\tau}(\mu \mathscr{V})\left(x_{2}\right)\right] \\
=\frac{1}{\kappa^{2} \Gamma_{\kappa}^{2}(\tau)} \int_{x_{1}}^{x_{2}}\left[\int_{x_{1}}^{\vartheta}\left(\Psi\left(x_{2}\right)-\Psi(\varrho)\right)^{\frac{\tau}{\kappa}-1} \Psi^{\prime}(\varrho) v(\varrho)\right. \\
\left.\quad \times \int_{x_{1}}^{x_{2}}\left(\Psi\left(x_{2}\right)-\Psi(\xi)\right)^{\frac{\tau}{\kappa}-1}(\xi-\varrho) \Psi^{\prime}(\xi) \mu(\xi) d \xi d \varrho\right]\left(\mathscr{V}^{\prime}(\vartheta)\right) d \vartheta \\
=\frac{1}{\kappa \Gamma_{\kappa}(\tau)} \int_{x_{1}}^{x_{2}}\left[\frac{1}{\kappa \Gamma_{\kappa}(\tau)} \int_{x_{1}}^{x_{2}}\left(\Psi\left(x_{2}\right)-\Psi(\xi)\right)^{\frac{\tau}{\kappa}-1} \Psi^{\prime}(\xi) \xi \mu(\xi) d \xi\right. \\
\quad \times \int_{x_{1}}^{\vartheta}\left(\Psi\left(x_{2}\right)-\Psi(\varrho)\right)^{\frac{\tau}{\kappa}-1} \Psi^{\prime}(\varrho) \nu(\varrho) d \varrho-\frac{1}{\kappa \Gamma_{\kappa}(\tau)} \\
\quad \times \int_{x_{1}}^{x_{2}}\left(\Psi\left(x_{2}\right)-\Psi(\xi)\right)^{\frac{\tau}{\kappa}-1} \Psi^{\prime}(\xi) \mu(\xi) d \xi \times \int_{x_{1}}^{\vartheta} \\
\left.\quad \times\left(\Psi\left(x_{2}\right)-\Psi(\varrho)\right)^{\frac{\tau}{\kappa}-1} \Psi^{\prime}(\varrho) \varrho v(\varrho) d \varrho\right]\left(\mathscr{V}^{\prime}(\vartheta)\right) d \vartheta .
\end{aligned}
$$


The latter by (9) gives

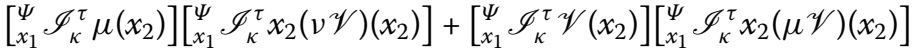

$$
\begin{aligned}
& -\left[{ }_{x_{1}}^{\Psi} \mathscr{I}_{\kappa}^{\tau} x_{2}(\mu)\left(x_{2}\right)\right]\left[{ }_{x_{1}}^{\Psi} \mathscr{I}_{\kappa}^{\tau}(v \mathscr{V})\left(x_{2}\right)\right]-\left[{ }_{x_{1}}^{\Psi} \mathscr{I}_{\kappa}^{\tau} x_{2}(v)\left(x_{2}\right)\right]\left[\begin{array}{c}
\Psi \\
x_{1}
\end{array} \mathscr{I}_{\kappa}^{\tau}(\mu \mathscr{V})\left(x_{2}\right)\right] \\
& =\frac{1}{\kappa \Gamma_{\kappa}(\tau)} \int_{x_{1}}^{x_{2}}\left[{ }_{x_{1}}^{\Psi} \mathscr{I}_{\kappa}^{\tau} x_{2} \mu\left(x_{2}\right) \int_{x_{1}}^{\vartheta}\left(\Psi\left(x_{2}\right)-\Psi(\varrho)\right)^{\frac{\tau}{\kappa}-1} \Psi^{\prime}(\varrho) \nu(\varrho) d \varrho\right. \\
& \left.-{ }_{x_{1}}^{\Psi} \mathscr{I}_{\kappa}^{\tau} \mu\left(x_{2}\right) \int_{x_{1}}^{\vartheta} \varrho\left(\Psi\left(x_{2}\right)-\Psi(\varrho)\right)^{\frac{\tau}{\kappa}-1} \Psi^{\prime}(\varrho) v(\varrho) d \varrho\right]\left(\mathscr{V}^{\prime}(\vartheta)\right) d \vartheta \text {, }
\end{aligned}
$$

which completes the proof.

Based on Lemma 3.1, we prove the following theorem.

Theorem 3.1 Let $\Psi$ be a measurable increasing positive function on $\left(x_{1}, x_{2}\right)$ with continuous derivative $\Psi^{\prime}(\varrho)$ on $\left[x_{1}, x_{2}\right]$. Let $\Phi:\left[x_{1}, x_{2}\right] \rightarrow \mathbb{R}$ be an absolutely continuous function with $\left(\Phi^{\prime}\right)^{2} \in L_{1}\left[x_{1}, x_{2}\right]$, and let $\mu, v:\left[x_{1}, x_{2}\right] \rightarrow \mathbb{R}^{+}$be positive integrable functions. Then for all $\tau, \kappa>0$, we have

$$
\begin{aligned}
& {\left[{ }_{x_{1}}^{\Psi} \mathscr{I}_{\kappa}^{\tau} \mu\left(x_{2}\right)\right]\left[{ }_{x_{1}}^{\Psi} \mathscr{I}_{\kappa}^{\tau}\left(v \Phi^{2}\right)\left(x_{2}\right)\right]+\left[{ }_{x_{1}}^{\Psi} \mathscr{I}_{\kappa}^{\tau} v\left(x_{2}\right)\right]\left[{ }_{x_{1}}^{\Psi} \mathscr{I}_{\kappa}^{\tau}\left(\mu \Phi^{2}\right)\left(x_{2}\right)\right]}
\end{aligned}
$$

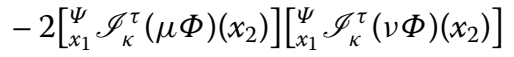

$$
\begin{aligned}
& \leq \frac{1}{\kappa \Gamma_{\kappa}(\tau)} \int_{x_{1}}^{x_{2}}\left[{ }_{x_{1}}^{\Psi} \mathscr{I}_{\kappa}^{\tau} x_{2} \mu\left(x_{2}\right) \int_{x_{1}}^{\vartheta}\left(\Psi\left(x_{2}\right)-\Psi(\varrho)\right)^{\frac{\tau}{\kappa}-1} \Psi^{\prime}(\varrho) v(\varrho) d \varrho\right. \\
& \left.-{ }_{x_{1}}^{\Psi} \mathscr{I}_{\kappa}^{\tau} \mu\left(x_{2}\right) \int_{x_{1}}^{\vartheta} \varrho\left(\Psi\left(x_{2}\right)-\Psi(\varrho)\right)^{\frac{\tau}{\kappa}-1} \Psi^{\prime}(\varrho) \nu(\varrho) d \varrho\right]\left(\Phi^{\prime}(\vartheta)\right)^{2} d \vartheta .
\end{aligned}
$$

Proof By employing definition (9) and Lemma 3.1 we obtain

$$
\begin{aligned}
{\left[{ }_{x_{1}}^{\Psi} \mathscr{I}_{\kappa}^{\tau} \mu\left(x_{2}\right)\right]\left[{ }_{x_{1}}^{\Psi} \mathscr{I}_{\kappa}^{\tau}\left(v \Phi^{2}\right)\left(x_{2}\right)\right]+\left[{ }_{x_{1}}^{\Psi} \mathscr{I}_{\kappa}^{\tau} v\left(x_{2}\right)\right]\left[{ }_{x_{1}}^{\Psi} \mathscr{I}_{\kappa}^{\tau}\left(\mu \Phi^{2}\right)\left(x_{2}\right)\right] } \\
\quad-2\left[{ }_{x_{1}}^{\Psi} \mathscr{I}_{\kappa}^{\tau}(\mu \Phi)\left(x_{2}\right)\right]\left[\begin{array}{l}
\Psi \\
x_{1}
\end{array} \mathscr{I}_{\kappa}^{\tau}(\nu \Phi)\left(x_{2}\right)\right] \\
=\frac{1}{\kappa^{2} \Gamma_{\kappa}^{2}(\tau)} \int_{x_{1}}^{x_{2}} \int_{x_{1}}^{x_{2}}\left(\Psi\left(x_{2}\right)-\Psi(\xi)\right)^{\frac{\tau}{\kappa}-1} \\
\quad \times\left(\Psi\left(x_{2}\right)-\Psi(\varrho)\right)^{\frac{\tau}{\kappa}-1} \Psi^{\prime}(\xi) \mu(\xi) \Psi^{\prime}(\varrho) v(\varrho)(\Phi(\xi)-\Phi(\varrho))^{2} d \xi d \varrho \\
=\frac{1}{\kappa^{2} \Gamma_{\kappa}^{2}(\tau)} \int_{x_{1}}^{x_{2}} \int_{x_{1}}^{x_{2}}\left(\Psi\left(x_{2}\right)-\Psi(\xi)\right)^{\frac{\tau}{\kappa}-1} \\
\quad \times\left(\Psi\left(x_{2}\right)-\Psi(\varrho)\right)^{\frac{\tau}{\kappa}-1} \Psi^{\prime}(\xi) \mu(\xi) \Psi^{\prime}(\varrho) v(\varrho)(\xi-\varrho)^{2}\left(\frac{\Phi(\xi)-\Phi(\varrho)}{\xi-\varrho}\right)^{2} d \xi d \varrho .
\end{aligned}
$$

Consequently, it follows that

$$
\begin{gathered}
{\left[{ }_{x_{1}}^{\Psi} \mathscr{I}_{\kappa}^{\tau} \mu\left(x_{2}\right)\right]\left[{ }_{x_{1}}^{\Psi} \mathscr{I}_{\kappa}^{\tau}\left(\nu \Phi^{2}\right)\left(x_{2}\right)\right]+\left[{ }_{x_{1}}^{\Psi} \mathscr{I}_{\kappa}^{\tau} \nu\left(x_{2}\right)\right]\left[{ }_{x_{1}}^{\Psi} \mathscr{I}_{\kappa}^{\tau}\left(\mu \Phi^{2}\right)\left(x_{2}\right)\right]} \\
-2\left[{ }_{x_{1}}^{\Psi} \mathscr{I}_{\kappa}^{\tau}(\mu \Phi)\left(x_{2}\right)\right]\left[{ }_{x_{1}}^{\Psi} \mathscr{I}_{\kappa}^{\tau}(\nu \Phi)\left(x_{2}\right)\right]
\end{gathered}
$$




$$
\begin{aligned}
\leq & \frac{1}{\kappa^{2} \Gamma_{\kappa}^{2}(\tau)} \int_{x_{1}}^{x_{2}} \int_{x_{1}}^{x_{2}}\left(\Psi\left(x_{2}\right)-\Psi(\xi)\right)^{\frac{\tau}{\kappa}-1} \\
& \times\left(\Psi\left(x_{2}\right)-\Psi(\varrho)\right)^{\frac{\tau}{\kappa}-1} \Psi^{\prime}(\xi) \mu(\xi) \Psi^{\prime}(\varrho) v(\varrho)(\xi-\varrho)^{2}\left(\frac{\int_{\varrho}^{\xi} \Phi^{\prime}(\vartheta) d \vartheta}{\xi-\varrho}\right)^{2} d \xi d \varrho .
\end{aligned}
$$

By the Cauchy-Schwarz inequality [11] we get

$$
\begin{aligned}
& {\left[{ }_{x_{1}}^{\Psi} \mathscr{I}_{\kappa}^{\tau} \mu\left(x_{2}\right)\right]\left[{ }_{x_{1}}^{\Psi} \mathscr{I}_{\kappa}^{\tau}\left(v \Phi^{2}\right)\left(x_{2}\right)\right]+\left[{ }_{x_{1}}^{\Psi} \mathscr{I}_{\kappa}^{\tau} v\left(x_{2}\right)\right]\left[{ }_{x_{1}}^{\Psi} \mathscr{I}_{\kappa}^{\tau}\left(\mu \Phi^{2}\right)\left(x_{2}\right)\right] } \\
& \quad-2\left[{ }_{x_{1}}^{\Psi} \mathscr{I}_{\kappa}^{\tau}(\mu \Phi)\left(x_{2}\right)\right]\left[{ }_{x_{1}}^{\Psi} \mathscr{I}_{\kappa}^{\tau}(v \Phi)\left(x_{2}\right)\right] \\
&=\frac{1}{\kappa^{2} \Gamma_{\kappa}^{2}(\tau)} \int_{x_{1}}^{x_{2}} \int_{x_{1}}^{x_{2}}\left(\Psi\left(x_{2}\right)-\Psi(\xi)\right)^{\frac{\tau}{\kappa}-1} \\
& \quad \times\left(\Psi\left(x_{2}\right)-\Psi(\varrho)\right)^{\frac{\tau}{\kappa}-1} \Psi^{\prime}(\xi) \mu(\xi) \Psi^{\prime}(\varrho) v(\varrho)(\xi-\varrho)^{2}\left(\frac{\int_{\varrho}^{\xi} \Phi^{\prime}(\vartheta) d \vartheta}{\xi-\varrho}\right)^{2} d \xi d \varrho \\
& \leq \frac{1}{\kappa^{2} \Gamma_{\kappa}^{2}(\tau)} \int_{x_{1}}^{x_{2}} \int_{x_{1}}^{x_{2}}\left(\Psi\left(x_{2}\right)-\Psi(\xi)\right)^{\frac{\tau}{\kappa}-1}\left(\Psi\left(x_{2}\right)-\Psi(\varrho)\right)^{\frac{\tau}{\kappa}-1} \\
& \times \Psi^{\prime}(\xi) \mu(\xi) \Psi^{\prime}(\varrho) v(\varrho)(\xi-\varrho)^{2}\left(\frac{\left(\int_{\varrho}^{\xi} d \vartheta\right)^{\frac{1}{2}}\left(\int_{\varrho}^{\xi}\left(\Phi^{\prime}(\vartheta)\right)^{2} d \vartheta\right)^{\frac{1}{2}}}{\xi-\varrho}\right)^{2} d \xi d \varrho \\
& \leq \frac{1}{\kappa^{2} \Gamma_{\kappa}^{2}(\tau)} \int_{x_{1}}^{x_{2}} \int_{x_{1}}^{x_{2}}\left(\Psi\left(x_{2}\right)-\Psi(\xi)\right)^{\frac{\tau}{\kappa}-1} \\
& \times\left(\Psi\left(x_{2}\right)-\Psi(\varrho)\right)^{\frac{\tau}{\kappa}-1} \Psi^{\prime}(\xi) \mu(\xi) \Psi^{\prime}(\varrho) \nu(\varrho)(\xi-\varrho)^{2}\left(\frac{\left(\int_{\varrho}^{\xi}\left(\Phi^{\prime}(\vartheta)\right)^{2} d \vartheta\right)}{\xi-\varrho}\right) d \xi d \varrho \\
& \leq \frac{1}{\kappa^{2} \Gamma_{\kappa}^{2}(\tau)} \int_{x_{1}}^{x_{2}} \int_{x_{1}}^{x_{2}}\left(\Psi\left(x_{2}\right)-\Psi(\xi)\right)^{\frac{\tau}{\kappa}-1} \\
& \times\left(\Psi\left(x_{2}\right)-\Psi(\varrho)\right)^{\frac{\tau}{\kappa}-1} \Psi^{\prime}(\xi) \mu(\xi) \Psi^{\prime}(\varrho) v(\varrho)(\xi-\varrho)\left(\int_{\varrho}^{\xi}\left(\Phi^{\prime}(\vartheta)\right)^{2} d \vartheta\right) d \xi d \varrho .
\end{aligned}
$$

Hence using (13) and (15), we conclude the proof.

Corollary 3.1 Let $\Psi$ be a measurable increasing positive function on $\left(x_{1}, x_{2}\right)$ with continuous derivative $\Psi^{\prime}(\varrho)$ on $\left[x_{1}, x_{2}\right]$. Let $\Phi:\left[x_{1}, x_{2}\right] \rightarrow \mathbb{R}$ be an absolutely continuous function with $\left(\Phi^{\prime}\right)^{2} \in L_{1}\left[x_{1}, x_{2}\right]$, and let $v:\left[x_{1}, x_{2}\right] \rightarrow \mathbb{R}^{+}$be positive integrable. Then for all $\tau, \kappa>0$, we have

$$
\begin{aligned}
& {\left[\frac{\left(\Psi\left(x_{2}\right)-\Psi\left(x_{1}\right)\right)^{\frac{\tau}{\kappa}}}{\Gamma_{\kappa}(\tau+\kappa)}\right]\left[{ }_{x_{1}}^{\Psi} \mathscr{I}_{\kappa}^{\tau}\left(v \Phi^{2}\right)\left(x_{2}\right)\right]+\left[{ }_{x_{1}}^{\Psi} \mathscr{I}_{\kappa}^{\tau} v\left(x_{2}\right)\right]\left[{ }_{x_{1}}^{\Psi} \mathscr{I}_{\kappa}^{\tau}\left(\Phi^{2}\right)\left(x_{2}\right)\right]} \\
& -2\left[{ }_{x_{1}}^{\Psi} \mathscr{I}_{\kappa}^{\tau}(\Phi)\left(x_{2}\right)\right]\left[\begin{array}{l}
\Psi \\
x_{1}
\end{array} \mathscr{I}_{\kappa}^{\tau}(v \Phi)\left(x_{2}\right)\right] \\
& \leq \frac{1}{\kappa \Gamma_{\kappa}(\tau)} \int_{x_{1}}^{x_{2}}\left[{ }_{x_{1}}^{\Psi} \mathscr{I}_{\kappa}^{\tau} x_{2} \int_{x_{1}}^{\vartheta}\left(\Psi\left(x_{2}\right)-\Psi(\varrho)\right)^{\frac{\tau}{\kappa}-1} \Psi^{\prime}(\varrho) v(\varrho) d \varrho\right. \\
& \left.-\frac{\left(\Psi\left(x_{2}\right)-\Psi\left(x_{1}\right)\right)^{\frac{\tau}{\kappa}}}{\Gamma_{\kappa}(\tau+\kappa)} \int_{x_{1}}^{\vartheta} \varrho\left(\Psi\left(x_{2}\right)-\Psi(\varrho)\right)^{\frac{\tau}{\kappa}-1} \Psi^{\prime}(\varrho) v(\varrho) d \varrho\right]\left(\Phi^{\prime}(\vartheta)\right)^{2} d \vartheta .
\end{aligned}
$$

Proof By considering $\mu(\vartheta)=1, \vartheta \in\left[x_{1}, x_{2}\right]$, in Theorem 3.1 we obtain the desired result. $\square$ 
Corollary 3.2 Let $\Psi$ be a measurable increasing positive function on $\left(x_{1}, x_{2}\right)$ with continuous derivative $\Psi^{\prime}(\varrho)$ on $\left[x_{1}, x_{2}\right]$. Let $\Phi:\left[x_{1}, x_{2}\right] \rightarrow \mathbb{R}$ be an absolutely continuous function with $\left(\Phi^{\prime}\right)^{2} \in L_{1}\left[x_{1}, x_{2}\right]$, and let $\mu:\left[x_{1}, x_{2}\right] \rightarrow \mathbb{R}^{+}$be positive integrable. Then for all $\tau, \kappa>0$, we have

$$
\begin{aligned}
{\left[{ }_{x_{1}}^{\Psi} \mathscr{I}_{\kappa}^{\tau} \mu\left(x_{2}\right)\right]\left[{ }_{x_{1}}^{\Psi} \mathscr{I}_{\kappa}^{\tau}\left(\Phi^{2}\right)\left(x_{2}\right)\right]+\left[\frac{\left(\Psi\left(x_{2}\right)-\Psi\left(x_{1}\right)\right)^{\frac{\tau}{\kappa}}}{\Gamma_{\kappa}(\tau+\kappa)}\right]\left[{ }_{x_{1}}^{\Psi} \mathscr{I}_{\kappa}^{\tau}\left(\Phi^{2}\right)\left(x_{2}\right)\right] } \\
\quad-2\left[{ }_{x_{1}}^{\Psi} \mathscr{I}_{\kappa}^{\tau}(\mu \Phi)\left(x_{2}\right)\right]\left[{ }_{x_{1}}^{\Psi} \mathscr{I}_{\kappa}^{\tau}(\Phi)\left(x_{2}\right)\right] \\
\leq \frac{1}{\kappa \Gamma_{\kappa}(\tau)} \int_{x_{1}}^{x_{2}}\left[{ }_{x_{1}}^{\Psi} \mathscr{I}_{\kappa}^{\tau} x_{2} \mu\left(x_{2}\right) \int_{x_{1}}^{\vartheta}\left(\Psi\left(x_{2}\right)-\Psi(\varrho)\right)^{\frac{\tau}{\kappa}-1} \Psi^{\prime}(\varrho) d \varrho\right. \\
\left.\quad-{ }_{x_{1}}^{\Psi} \mathscr{I}_{\kappa}^{\tau} \mu\left(x_{2}\right) \int_{x_{1}}^{\vartheta} \varrho\left(\Psi\left(x_{2}\right)-\Psi(\varrho)\right)^{\frac{\tau}{\kappa}-1} \Psi^{\prime}(\varrho) d \varrho\right]\left(\Phi^{\prime}(\vartheta)\right)^{2} d \vartheta .
\end{aligned}
$$

Proof By considering $v(\vartheta)=1, \vartheta \in\left[x_{1}, x_{2}\right]$, in Theorem 3.1 we get the desired result.

Corollary 3.3 Let $\Psi$ be a measurable increasing positive function on $\left(x_{1}, x_{2}\right)$ with continuous derivative $\Psi^{\prime}(\varrho)$ on $\left[x_{1}, x_{2}\right]$. Let $\Phi:\left[x_{1}, x_{2}\right] \rightarrow \mathbb{R}$ be an absolutely continuous function with $\left(\Phi^{\prime}\right)^{2} \in L_{1}\left[x_{1}, x_{2}\right]$. Then for all $\tau, \kappa>0$, we have

$$
\begin{aligned}
& {\left[\frac{\left(\Psi\left(x_{2}\right)-\Psi\left(x_{1}\right)\right)^{\frac{\tau}{\kappa}}}{\Gamma_{\kappa}(\tau+\kappa)}\right]\left[{ }_{x_{1}}^{\Psi} \mathscr{I}_{\kappa}^{\tau}\left(\Phi^{2}\right)\left(x_{2}\right)\right]-\left[{ }_{x_{1}}^{\Psi} \mathscr{I}_{\kappa}^{\tau}(\Phi)\left(x_{2}\right)\right]^{2}} \\
& \leq \frac{1}{2 \kappa \Gamma_{\kappa}(\tau)} \int_{x_{1}}^{x_{2}}\left[\begin{array}{l}
\Psi \\
x_{1}
\end{array} \mathscr{J}_{\kappa}^{\tau} x_{2} \int_{x_{1}}^{\vartheta}\left(\Psi\left(x_{2}\right)-\Psi(\varrho)\right)^{\frac{\tau}{\kappa}-1} \Psi^{\prime}(\varrho) d \varrho\right. \\
& \left.\quad-\frac{\left(\Psi\left(x_{2}\right)-\Psi\left(x_{1}\right)\right)^{\frac{\tau}{\kappa}}}{\Gamma_{\kappa}(\tau+\kappa)} \int_{x_{1}}^{\vartheta} \varrho\left(\Psi\left(x_{2}\right)-\Psi(\varrho)\right)^{\frac{\tau}{\kappa}-1} \Psi^{\prime}(\varrho) d \varrho\right]\left(\Phi^{\prime}(\vartheta)\right)^{2} d \vartheta .
\end{aligned}
$$

Proof Taking $\mu(\vartheta)=v(\vartheta)=1, \vartheta \in\left[x_{1}, x_{2}\right]$, in Theorem 3.1, we obtain the desired result.

Theorem 3.2 Let $\Psi$ be a measurable increasing positive function on $\left(x_{1}, x_{2}\right)$ with continuous derivative $\Psi^{\prime}(\varrho)$ on $\left[x_{1}, x_{2}\right]$. Let $f_{1}, f_{2}:\left[x_{1}, x_{2}\right] \rightarrow \mathbb{R}$ be absolutely continuous functions with $\left(f_{1}^{\prime}\right)^{2} \in L_{1}\left[x_{1}, x_{2}\right]$ and $\left(f_{2}^{\prime}\right)^{2} \in L_{1}\left[x_{1}, x_{2}\right]$, and let $\mu, v:\left[x_{1}, x_{2}\right] \rightarrow \mathbb{R}^{+}$be positive integrable. Then for all $\tau, \kappa>0$, we have

$$
\begin{aligned}
\left.\right|_{x_{1}} ^{\Psi} \mathscr{I}_{\kappa}^{\tau} \mu\left(x_{2}\right)_{x_{1}}^{\Psi} \mathscr{I}_{\kappa}^{\tau}\left(v f_{1} f_{2}\right)\left(x_{2}\right)+{ }_{x_{1}}^{\Psi} \mathscr{I}_{\kappa}^{\tau} v\left(x_{2}\right)_{x_{1}}^{\Psi} \mathscr{I}_{\kappa}^{\tau}\left(\mu f_{1} f_{2}\right)\left(x_{2}\right) \\
\quad-{ }_{x_{1}}^{\Psi} \mathscr{I}_{\kappa}^{\tau}\left(v f_{1}\right)\left(x_{2}\right)_{x_{1}}^{\Psi} \mathscr{I}_{\kappa}^{\tau}\left(\mu f_{2}\right)\left(x_{2}\right)-{ }_{x_{1}}^{\Psi} \mathscr{I}_{\kappa}^{\tau}\left(\mu f_{1}\right)\left(x_{2}\right)_{x_{1}}^{\Psi} \mathscr{I}_{\kappa}^{\tau}\left(v f_{2}\right)\left(x_{2}\right) \mid \\
\leq \frac{1}{\kappa \Gamma_{\kappa}(\tau)}\left(\int _ { x _ { 1 } } ^ { x _ { 2 } } \left[{ }_{x_{1}}^{\Psi} \mathscr{I}_{\kappa}^{\tau} x_{2} \mu\left(x_{2}\right) \int_{x_{1}}^{\vartheta}\left(\Psi\left(x_{2}\right)-\Psi(\varrho)\right)^{\frac{\tau}{\kappa}-1} \Psi^{\prime}(\varrho) v(\varrho) d \varrho\right.\right. \\
\left.\left.\quad-{ }_{x_{1}}^{\Psi} \mathscr{I}_{\kappa}^{\tau} \mu\left(x_{2}\right) \int_{x_{1}}^{\vartheta} \varrho\left(\Psi\left(x_{2}\right)-\Psi(\varrho)\right)^{\frac{\tau}{\kappa}-1} \Psi^{\prime}(\varrho) v(\varrho) d \varrho\right]\left(f_{1}^{\prime}(\vartheta)\right)^{2} d \vartheta\right)^{\frac{1}{2}} \\
\quad \times\left(\int _ { x _ { 1 } } ^ { x _ { 2 } } \left[{ }_{x_{1}}^{\Psi} \mathscr{I}_{\kappa}^{\tau} x_{2} \mu\left(x_{2}\right) \int_{x_{1}}^{\vartheta}\left(\Psi\left(x_{2}\right)-\Psi(\varrho)\right)^{\frac{\tau}{\kappa}-1} \Psi^{\prime}(\varrho) v(\varrho) d \varrho\right.\right. \\
\left.\left.\quad-{ }_{x_{1}}^{\Psi} \mathscr{I}_{\kappa}^{\tau} \mu\left(x_{2}\right) \int_{x_{1}}^{\vartheta} \varrho\left(\Psi\left(x_{2}\right)-\Psi(\varrho)\right)^{\frac{\tau}{\kappa}-1} \Psi^{\prime}(\varrho) v(\varrho) d \varrho\right]\left(f_{2}^{\prime}(\vartheta)\right)^{2} d \vartheta\right)^{\frac{1}{2}} .
\end{aligned}
$$


Proof Considering the left-hand side of (16), we have

$$
\begin{aligned}
& \left.\right|_{x_{1}} ^{\Psi} \mathscr{I}_{\kappa}^{\tau} \mu\left(x_{2}\right)_{x_{1}}^{\Psi} \mathscr{I}_{\kappa}^{\tau}\left(\nu f_{1} f_{2}\right)\left(x_{2}\right)+{ }_{x_{1}}^{\Psi} \mathscr{I}_{\kappa}^{\tau} \nu\left(x_{2}\right)_{x_{1}}^{\Psi} \mathscr{I}_{\kappa}^{\tau}\left(\mu f_{1} f_{2}\right)\left(x_{2}\right) \\
& -{ }_{x_{1}}^{\Psi} \mathscr{I}_{\kappa}^{\tau}\left(v f_{1}\right)\left(x_{2}\right)_{x_{1}}^{\Psi} \mathscr{I}_{\kappa}^{\tau}\left(\mu f_{2}\right)\left(x_{2}\right)-{ }_{x_{1}}^{\Psi} \mathscr{I}_{\kappa}^{\tau}\left(\mu f_{1}\right)\left(x_{2}\right)_{x_{1}}^{\Psi} \mathscr{I}_{\kappa}^{\tau}\left(v f_{2}\right)\left(x_{2}\right) \mid \\
& \leq \frac{1}{\kappa^{2} \Gamma_{\kappa}^{2}(\tau)}\left(\int_{x_{1}}^{x_{2}} \int_{x_{1}}^{x_{2}}\left(\Psi\left(x_{2}\right)-\Psi(\xi)\right)^{\frac{\tau}{\kappa}-1}\left(\Psi\left(x_{2}\right)-\Psi(\varrho)\right)^{\frac{\tau}{\kappa}-1} \Psi^{\prime}(\xi) \mu(\xi)\right. \\
& \left.\times \Psi^{\prime}(\varrho) v(\varrho)\left(f_{1}(\xi)-f_{1}(\varrho)\right)^{2} d \xi d \varrho\right)^{\frac{1}{2}} \times\left(\int_{x_{1}}^{x_{2}} \int_{x_{1}}^{x_{2}}\left(\Psi\left(x_{2}\right)-\Psi(\xi)\right)^{\frac{\tau}{\kappa}-1}\right. \\
& \left.\times\left(\Psi\left(x_{2}\right)-\Psi(\varrho)\right)^{\frac{\tau}{\kappa}-1} \Psi^{\prime}(\xi) \mu(\xi) \Psi^{\prime}(\varrho) \nu(\varrho)\left(f_{2}(\xi)-f_{2}(\varrho)\right)^{2} d \xi d \varrho\right)^{\frac{1}{2}} \\
& \leq \frac{1}{\kappa^{2} \Gamma_{\kappa}^{2}(\tau)}\left(\int_{x_{1}}^{x_{2}} \int_{x_{1}}^{x_{2}}\left(\Psi\left(x_{2}\right)-\Psi(\xi)\right)^{\frac{\tau}{\kappa}-1}\left(\Psi\left(x_{2}\right)-\Psi(\varrho)\right)^{\frac{\tau}{\kappa}-1} \Psi^{\prime}(\xi) \mu(\xi)\right. \\
& \left.\times \Psi^{\prime}(\varrho) v(\varrho)\left(\int_{\varrho}^{\xi} f_{1}^{\prime}(\vartheta) d \vartheta\right)^{2} d \xi d \varrho\right)^{\frac{1}{2}} \times\left(\int_{x_{1}}^{x_{2}} \int_{x_{1}}^{x_{2}}\left(\Psi\left(x_{2}\right)-\Psi(\xi)\right)^{\frac{\tau}{\kappa}-1}\right. \\
& \left.\times\left(\Psi\left(x_{2}\right)-\Psi(\varrho)\right)^{\frac{\tau}{\kappa}-1} \Psi^{\prime}(\xi) \mu(\xi) \Psi^{\prime}(\varrho) \nu(\varrho)\left(\int_{\varrho}^{\xi} f_{2}^{\prime}(\vartheta) d \vartheta\right)^{2} d \xi d \varrho\right)^{\frac{1}{2}}
\end{aligned}
$$

Applying the Cauchy-Schwarz inequality [11] to this inequality, we get

$$
\begin{aligned}
& \left.\right|_{x_{1}} ^{\Psi} \mathscr{I}_{\kappa}^{\tau} \mu\left(x_{2}\right)_{x_{1}}^{\Psi} \mathscr{I}_{\kappa}^{\tau}\left(\nu f_{1} f_{2}\right)\left(x_{2}\right)+{ }_{x_{1}}^{\Psi} \mathscr{I}_{\kappa}^{\tau} v\left(x_{2}\right)_{x_{1}}^{\Psi} \mathscr{I}_{\kappa}^{\tau}\left(\mu f_{1} f_{2}\right)\left(x_{2}\right) \\
& -{ }_{x_{1}}^{\Psi} \mathscr{I}_{\kappa}^{\tau}\left(v f_{1}\right)\left(x_{2}\right)_{x_{1}}^{\Psi} \mathscr{I}_{\kappa}^{\tau}\left(\mu f_{2}\right)\left(x_{2}\right)-{ }_{x_{1}}^{\Psi} \mathscr{I}_{\kappa}^{\tau}\left(\mu f_{1}\right)\left(x_{2}\right)_{x_{1}}^{\Psi} \mathscr{I}_{\kappa}^{\tau}\left(v f_{2}\right)\left(x_{2}\right) \mid \\
& \leq \frac{1}{\kappa^{2} \Gamma_{\kappa}^{2}(\tau)}\left[\int_{x_{1}}^{x_{2}} \int_{x_{1}}^{x_{2}}\left(\Psi\left(x_{2}\right)-\Psi(\xi)\right)^{\frac{\tau}{\kappa}-1}\left(\Psi\left(x_{2}\right)-\Psi(\varrho)\right)^{\frac{\tau}{\kappa}-1} \Psi^{\prime}(\xi) \mu(\xi)\right. \\
& \left.\times \Psi^{\prime}(\varrho) v(\varrho)\left(\left(\int_{\varrho}^{\xi} d \vartheta\right)^{\frac{1}{2}}\left(\int_{\varrho}^{\xi}\left(f_{1}^{\prime}(\vartheta)\right)^{2} d \vartheta\right)^{\frac{1}{2}}\right)^{2} d \xi d \varrho\right]^{\frac{1}{2}} \\
& \times\left[\int_{x_{1}}^{x_{2}} \int_{x_{1}}^{x_{2}}\left(\Psi\left(x_{2}\right)-\Psi(\xi)\right)^{\frac{\tau}{\kappa}-1}\left(\Psi\left(x_{2}\right)-\Psi(\varrho)\right)^{\frac{\tau}{\kappa}-1}\right. \\
& \left.\times \Psi^{\prime}(\xi) \mu(\xi) \Psi^{\prime}(\varrho) \nu(\varrho)\left(\left(\int_{\varrho}^{\xi} d \vartheta\right)^{\frac{1}{2}}\left(\int_{\varrho}^{\xi}\left(f_{2}^{\prime}(\vartheta)\right)^{2} d \vartheta\right)^{\frac{1}{2}}\right)^{2} d \xi d \varrho\right]^{\frac{1}{2}} \\
& \leq \frac{1}{\kappa^{2} \Gamma_{\kappa}^{2}(\tau)}\left[\int_{x_{1}}^{x_{2}} \int_{x_{1}}^{x_{2}}\left(\Psi\left(x_{2}\right)-\Psi(\xi)\right)^{\frac{\tau}{\kappa}-1}\left(\Psi\left(x_{2}\right)-\Psi(\varrho)\right)^{\frac{\tau}{\kappa}-1} \Psi^{\prime}(\xi) \mu(\xi)\right. \\
& \left.\times \Psi^{\prime}(\varrho) v(\varrho)(\xi-\varrho)\left(\int_{\varrho}^{\xi}\left(f_{1}^{\prime}(\vartheta)\right)^{2} d \vartheta\right) d \xi d \varrho\right]^{\frac{1}{2}} \times\left[\int_{x_{1}}^{x_{2}} \int_{x_{1}}^{x_{2}}\left(\Psi\left(x_{2}\right)-\Psi(\xi)\right)^{\frac{\tau}{\kappa}-1}\right. \\
& \left.\times\left(\Psi\left(x_{2}\right)-\Psi(\varrho)\right)^{\frac{\tau}{\kappa}-1} \Psi^{\prime}(\xi) \mu(\xi) \Psi^{\prime}(\varrho) v(\varrho)(\xi-\varrho)\left(\int_{\varrho}^{\xi}\left(f_{2}^{\prime}(\vartheta)\right)^{2} d \vartheta\right) d \xi d \varrho\right]^{\frac{1}{2}} \\
& \leq \frac{1}{\kappa^{2} \Gamma_{\kappa}^{2}(\tau)}\left[\int _ { x _ { 1 } } ^ { x _ { 2 } } \left(\int_{x_{1}}^{x_{2}} \xi\left(\Psi\left(x_{2}\right)-\Psi(\xi)\right)^{\frac{\tau}{\kappa}-1} \Psi^{\prime}(\xi) \mu(\xi) d \xi\right.\right. \\
& \left.\left.\times \int_{x_{1}}^{\vartheta}\left(\Psi\left(x_{2}\right)-\Psi(\varrho)\right)^{\frac{\tau}{\kappa}-1} \Psi^{\prime}(\varrho) v(\varrho) d \varrho\right)\left(f_{1}^{\prime}(\vartheta)\right)^{2}\right]^{\frac{1}{2}}
\end{aligned}
$$




$$
\begin{aligned}
& -\left[\int _ { x _ { 1 } } ^ { x _ { 2 } } \left(\int_{x_{1}}^{x_{2}}\left(\Psi\left(x_{2}\right)-\Psi(\xi)\right)^{\frac{\tau}{\kappa}-1} \Psi^{\prime}(\xi) \mu(\xi) d \xi\right.\right. \\
& \left.\left.\times \int_{x_{1}}^{\varrho} \varrho\left(\Psi\left(x_{2}\right)-\Psi(\varrho)\right)^{\frac{\tau}{\kappa}-1} \Psi^{\prime}(\varrho) v(\varrho) d \varrho\right)\left(f_{2}^{\prime}(\vartheta)\right)^{2}\right]^{\frac{1}{2}}
\end{aligned}
$$

In view of (9), we get the desired proof of (16).

Corollary 3.4 Let $\Psi$ be a measurable increasing positive function on $\left(x_{1}, x_{2}\right)$ with continuous derivative $\Psi^{\prime}(\varrho)$ on $\left[x_{1}, x_{2}\right]$. Let $f_{1}, f_{2}:\left[x_{1}, x_{2}\right] \rightarrow \mathbb{R}$ be absolutely continuous functions with $\left(f_{1}^{\prime}\right)^{2} \in L_{1}\left[x_{1}, x_{2}\right]$ and $\left(f_{2}^{\prime}\right)^{2} \in L_{1}\left[x_{1}, x_{2}\right]$, and let $v:\left[x_{1}, x_{2}\right] \rightarrow \mathbb{R}^{+}$be positive integrable. Then for all $\tau, \kappa>0$, we have

$$
\begin{aligned}
& \mid \frac{\left(\Psi\left(x_{2}\right)-\Psi\left(x_{1}\right)\right)^{\frac{\tau}{\kappa}}}{\Gamma_{\kappa}(\tau+\kappa)}{ }_{x_{1}} \mathscr{I}_{\kappa}^{\tau}\left(v f_{1} f_{2}\right)\left(x_{2}\right)+{ }_{x_{1}}^{\Psi} \mathscr{I}_{\kappa}^{\tau} v\left(x_{2}\right)_{x_{1}}^{\Psi} \mathscr{I}_{\kappa}^{\tau}\left(f_{1} f_{2}\right)\left(x_{2}\right) \\
& \quad-{ }_{x_{1}}^{\Psi} \mathscr{I}_{\kappa}^{\tau}\left(v f_{1}\right)\left(x_{2}\right)_{x_{1}}^{\Psi} \mathscr{I}_{\kappa}^{\tau}\left(f_{2}\right)\left(x_{2}\right)-{ }_{x_{1}}^{\Psi} \mathscr{I}_{\kappa}^{\tau}\left(f_{1}\right)\left(x_{2}\right)_{x_{1}}^{\Psi} \mathscr{I}_{\kappa}^{\tau}\left(v f_{2}\right)\left(x_{2}\right) \mid \\
& \leq \frac{1}{\kappa \Gamma_{\kappa}(\tau)}\left(\int _ { x _ { 1 } } ^ { x _ { 2 } } \left[{ }_{x_{1}}^{\Psi} \mathscr{I}_{\kappa}^{\tau} x_{2} \int_{x_{1}}^{\vartheta}\left(\Psi\left(x_{2}\right)-\Psi(\varrho)\right)^{\frac{\tau}{\kappa}-1} \Psi^{\prime}(\varrho) v(\varrho) d \varrho\right.\right. \\
& \left.\left.\quad-\frac{\left(\Psi\left(x_{2}\right)-\Psi\left(x_{1}\right)\right)^{\frac{\tau}{\kappa}}}{\Gamma_{\kappa}(\tau+\kappa)} \int_{x_{1}}^{\vartheta} \varrho\left(\Psi\left(x_{2}\right)-\Psi(\varrho)\right)^{\frac{\tau}{\kappa}-1} \Psi^{\prime}(\varrho) v(\varrho) d \varrho\right]\left(f_{1}^{\prime}(\vartheta)\right)^{2} d \vartheta\right)^{\frac{1}{2}} \\
& \quad \times\left(\int _ { x _ { 1 } } ^ { x _ { 2 } } \left[\begin{array}{l}
\Psi \\
x_{1}
\end{array} \mathscr{I}_{\kappa}^{\tau} x_{2} \int_{x_{1}}^{\vartheta}\left(\Psi\left(x_{2}\right)-\Psi(\varrho)\right)^{\frac{\tau}{\kappa}-1} \Psi^{\prime}(\varrho) v(\varrho) d \varrho\right.\right. \\
& \left.\left.\quad-\frac{\left(\Psi\left(x_{2}\right)-\Psi\left(x_{1}\right)\right)^{\frac{\tau}{\kappa}}}{\Gamma_{\kappa}(\tau+\kappa)} \int_{x_{1}}^{\vartheta} \varrho\left(\Psi\left(x_{2}\right)-\Psi(\varrho)\right)^{\frac{\tau}{\kappa}-1} \Psi^{\prime}(\varrho) v(\varrho) d \varrho\right]\left(f_{2}^{\prime}(\vartheta)\right)^{2} d \vartheta\right)^{\frac{1}{2}} .
\end{aligned}
$$

Proof Applying Theorem 3.2 with $\mu(\vartheta)=1, \vartheta \in\left[x_{1}, x_{2}\right]$, we obtain the desired result.

Corollary 3.5 Let $\Psi$ be a measurable increasing positive function on $\left(x_{1}, x_{2}\right)$ with continuous derivative $\Psi^{\prime}(\varrho)$ on $\left[x_{1}, x_{2}\right]$. Let $f_{1}, f_{2}:\left[x_{1}, x_{2}\right] \rightarrow \mathbb{R}$ be absolutely continuous functions with $\left(f_{1}^{\prime}\right)^{2} \in L_{1}\left[x_{1}, x_{2}\right]$ and $\left(f_{2}^{\prime}\right)^{2} \in L_{1}\left[x_{1}, x_{2}\right]$, and let $\mu:\left[x_{1}, x_{2}\right] \rightarrow \mathbb{R}^{+}$be positive integrable. Then for all $\tau, \kappa>0$, we have

$$
\begin{aligned}
& \mid x_{x_{1}} \mathscr{I}_{\kappa}^{\tau} \mu\left(x_{2}\right)_{x_{1}}^{\Psi} \mathscr{I}_{\kappa}^{\tau}\left(f_{1} f_{2}\right)\left(x_{2}\right)+\frac{\left(\Psi\left(x_{2}\right)-\Psi\left(x_{1}\right)\right)^{\frac{\tau}{\kappa}}}{\Gamma_{\kappa}(\tau+\kappa)} \underset{x_{1}}{\Psi} \mathscr{I}_{\kappa}^{\tau}\left(\mu f_{1} f_{2}\right)\left(x_{2}\right) \\
& -{ }_{x_{1}}^{\Psi} \mathscr{I}_{\kappa}^{\tau}\left(f_{1}\right)\left(x_{2}\right)_{x_{1}}^{\Psi} \mathscr{I}_{\kappa}^{\tau}\left(\mu f_{2}\right)\left(x_{2}\right)-{ }_{x_{1}}^{\Psi} \mathscr{I}_{\kappa}^{\tau}\left(\mu f_{1}\right)\left(x_{2}\right)_{x_{1}}^{\Psi} \mathscr{I}_{\kappa}^{\tau}\left(f_{2}\right)\left(x_{2}\right) \\
& \leq \frac{1}{\kappa \Gamma_{\kappa}(\tau)}\left(\int _ { x _ { 1 } } ^ { x _ { 2 } } \left[{ }_{x_{1}}^{\Psi} \mathscr{I}_{\kappa}^{\tau} x_{2} \mu\left(x_{2}\right) \int_{x_{1}}^{\vartheta}\left(\Psi\left(x_{2}\right)-\Psi(\varrho)\right)^{\frac{\tau}{\kappa}-1} \Psi^{\prime}(\varrho) d \varrho\right.\right. \\
& \left.\left.-{ }_{x_{1}}^{\Psi} \mathscr{I}_{\kappa}^{\tau} \mu\left(x_{2}\right) \int_{x_{1}}^{\vartheta} \varrho\left(\Psi\left(x_{2}\right)-\Psi(\varrho)\right)^{\frac{\tau}{\kappa}-1} \Psi^{\prime}(\varrho) d \varrho\right]\left(f_{1}^{\prime}(\vartheta)\right)^{2} d \vartheta\right)^{\frac{1}{2}} \\
& \times\left(\int _ { x _ { 1 } } ^ { x _ { 2 } } \left[\begin{array}{l}
\Psi \\
x_{1}
\end{array} \mathscr{I}_{\kappa}^{\tau} x_{2} \mu\left(x_{2}\right) \int_{x_{1}}^{\vartheta}\left(\Psi\left(x_{2}\right)-\Psi(\varrho)\right)^{\frac{\tau}{\kappa}-1} \Psi^{\prime}(\varrho) d \varrho\right.\right. \\
& \left.\left.-{ }_{x_{1}}^{\Psi} \mathscr{I}_{\kappa}^{\tau} \mu\left(x_{2}\right) \int_{x_{1}}^{\vartheta} \varrho\left(\Psi\left(x_{2}\right)-\Psi(\varrho)\right)^{\frac{\tau}{\kappa}-1} \Psi^{\prime}(\varrho) d \varrho\right]\left(f_{2}^{\prime}(\vartheta)\right)^{2} d \vartheta\right)^{\frac{1}{2}} \text {. }
\end{aligned}
$$


Proof Applying Theorem 3.2 with $v(\vartheta)=1, \vartheta \in\left[x_{1}, x_{2}\right]$, we obtain the desired result.

Corollary 3.6 Let $\Psi$ be a measurable increasing positive function on $\left(x_{1}, x_{2}\right)$ with continuous derivative $\Psi^{\prime}(\varrho)$ on $\left[x_{1}, x_{2}\right]$. Let $f_{1}, f_{2}:\left[x_{1}, x_{2}\right] \rightarrow \mathbb{R}$ be absolutely continuous functions with $\left(f_{1}^{\prime}\right)^{2} \in L_{1}\left[x_{1}, x_{2}\right]$ and $\left(f_{2}^{\prime}\right)^{2} \in L_{1}\left[x_{1}, x_{2}\right]$, and let $\mu:\left[x_{1}, x_{2}\right] \rightarrow \mathbb{R}^{+}$be positive integrable.

Then for all $\tau, \kappa>0$, we have

$$
\begin{aligned}
& \mid \frac{\left(\Psi\left(x_{2}\right)-\Psi\left(x_{1}\right)\right)^{\frac{\tau}{\kappa}}}{\Gamma_{\kappa}(\tau+\kappa)}{ }_{x_{1}} \mathscr{I}_{\kappa}^{\tau}\left(f_{1} f_{2}\right)\left(x_{2}\right)-{ }_{x_{1}}^{\Psi} \mathscr{I}_{\kappa}^{\tau}\left(f_{1}\right)\left(x_{2}\right)_{x_{1}}^{\Psi} \mathscr{I}_{\kappa}^{\tau}\left(\mu f_{2}\right)\left(x_{2}\right) \\
& -{ }_{x_{1}}^{\Psi} \mathscr{I}_{\kappa}^{\tau}\left(f_{1}\right)\left(x_{2}\right)_{x_{1}}^{\Psi} \mathscr{I}_{\kappa}^{\tau}\left(f_{2}\right)\left(x_{2}\right) \\
& \leq \frac{1}{2 \kappa \Gamma_{\kappa}(\tau)}\left(\int _ { x _ { 1 } } ^ { x _ { 2 } } \left[\begin{array}{l}
\Psi \\
x_{1}
\end{array} \mathscr{I}_{\kappa}^{\tau} x_{2} \int_{x_{1}}^{\vartheta}\left(\Psi\left(x_{2}\right)-\Psi(\varrho)\right)^{\frac{\tau}{\kappa}-1} \Psi^{\prime}(\varrho) d \varrho\right.\right. \\
& \left.\left.-\frac{\left(\Psi\left(x_{2}\right)-\Psi\left(x_{1}\right)\right)^{\frac{\tau}{\kappa}}}{\Gamma_{\kappa}(\tau+\kappa)} \int_{x_{1}}^{\vartheta} \varrho\left(\Psi\left(x_{2}\right)-\Psi(\varrho)\right)^{\frac{\tau}{\kappa}-1} \Psi^{\prime}(\varrho) d \varrho\right]\left(f_{1}^{\prime}(\vartheta)\right)^{2} d \vartheta\right)^{\frac{1}{2}} \\
& \times\left(\int _ { x _ { 1 } } ^ { x _ { 2 } } \left[{ }_{x_{1}}^{\Psi} \mathscr{I}_{\kappa}^{\tau} x_{2} \int_{x_{1}}^{\vartheta}\left(\Psi\left(x_{2}\right)-\Psi(\varrho)\right)^{\frac{\tau}{\kappa}-1} \Psi^{\prime}(\varrho) d \varrho\right.\right. \\
& \left.\left.-\frac{\left(\Psi\left(x_{2}\right)-\Psi\left(x_{1}\right)\right)^{\frac{\tau}{\kappa}}}{\Gamma_{\kappa}(\tau+\kappa)} \int_{x_{1}}^{\vartheta} \varrho\left(\Psi\left(x_{2}\right)-\Psi(\varrho)\right)^{\frac{\tau}{\kappa}-1} \Psi^{\prime}(\varrho) d \varrho\right]\left(f_{2}^{\prime}(\vartheta)\right)^{2} d \vartheta\right)^{\frac{1}{2}} .
\end{aligned}
$$

Proof Applying Theorem 3.2 with $\mu(\vartheta)=v(\vartheta)=1, \vartheta \in\left[x_{1}, x_{2}\right]$, we obtain the desired result.

Theorem 3.3 Let $\Psi$ be measurable increasing positive function on $\left(x_{1}, x_{2}\right)$ with continuous derivative $\Psi^{\prime}(\varrho)$ on $\left[x_{1}, x_{2}\right]$. Let $f_{1}:\left[x_{1}, x_{2}\right] \rightarrow \mathbb{R}$ be an absolutely continuous function with $\left(f_{1}^{\prime}\right) \in L^{\infty}\left[x_{1}, x_{2}\right]$, and let $f_{2}:\left[x_{1}, x_{2}\right] \rightarrow \mathbb{R}$ be nondecreasing. Moreover, let $\mu, v:\left[x_{1}, x_{2}\right] \rightarrow$ $\mathbb{R}^{+}$be positive integrable, Then for all $\tau, \kappa>0$, we have

$$
\begin{aligned}
\mid{ }_{x_{1}}^{\Psi} \mathscr{I}_{\kappa}^{\tau} \mu\left(x_{2}\right)_{x_{1}}^{\Psi} \mathscr{I}_{\kappa}^{\tau}\left(v f_{1} f_{2}\right)\left(x_{2}\right)+{ }_{x_{1}}^{\Psi} \mathscr{I}_{\kappa}^{\tau} v\left(x_{2}\right)_{x_{1}}^{\Psi} \mathscr{I}_{\kappa}^{\tau}\left(\mu f_{1} f_{2}\right)\left(x_{2}\right) \\
\quad-{ }_{x_{1}}^{\Psi} \mathscr{I}_{\kappa}^{\tau}\left(v f_{1}\right)\left(x_{2}\right)_{x_{1}}^{\Psi} \mathscr{I}_{\kappa}^{\tau}\left(\mu f_{2}\right)\left(x_{2}\right)-{ }_{x_{1}}^{\Psi} \mathscr{I}_{\kappa}^{\tau}\left(\mu f_{1}\right)\left(x_{2}\right)_{x_{1}}^{\Psi} \mathscr{I}_{\kappa}^{\tau}\left(v f_{2}\right)\left(x_{2}\right) \mid \\
\leq \frac{\left\|f_{1}^{\prime}\right\|_{\infty}}{\kappa \Gamma_{\kappa}(\tau)}\left[{ }_{x_{1}}^{\Psi} \mathscr{I}_{\kappa}^{\tau} x_{2} \mu\left(x_{2}\right) \int_{x_{1}}^{\vartheta}\left(\Psi\left(x_{2}\right)-\Psi(\varrho)\right)^{\frac{\tau}{\kappa}-1} \Psi^{\prime}(\varrho) v(\varrho) d \varrho\right. \\
\left.\quad-{ }_{x_{1}}^{\Psi} \mathscr{I}_{\kappa}^{\tau} \mu\left(x_{2}\right) \int_{x_{1}}^{\vartheta} \varrho\left(\Psi\left(x_{2}\right)-\Psi(\varrho)\right)^{\frac{\tau}{\kappa}-1} \Psi^{\prime}(\varrho) v(\varrho) d \varrho\right] \int_{x_{1}}^{x_{2}} f_{2}^{\prime}(\vartheta) d \vartheta .
\end{aligned}
$$

Proof Considering the left-hand side of (17), we have

$$
\begin{aligned}
\mid{ }_{x_{1}}^{\Psi} & \mathscr{I}_{\kappa}^{\tau} \mu\left(x_{2}\right)_{x_{1}}^{\Psi} \mathscr{I}_{\kappa}^{\tau}\left(v f_{1} f_{2}\right)\left(x_{2}\right)+{ }_{x_{1}}^{\Psi} \mathscr{I}_{\kappa}^{\tau} v\left(x_{2}\right)_{x_{1}}^{\Psi} \mathscr{I}_{\kappa}^{\tau}\left(\mu f_{1} f_{2}\right)\left(x_{2}\right) \\
& -{ }_{x_{1}}^{\Psi} \mathscr{J}_{\kappa}^{\tau}\left(v f_{1}\right)\left(x_{2}\right)_{x_{1}}^{\Psi} \mathscr{J}_{\kappa}^{\tau}\left(\mu f_{2}\right)\left(x_{2}\right)-{ }_{x_{1}}^{\Psi} \mathscr{I}_{\kappa}^{\tau}\left(\mu f_{1}\right)\left(x_{2}\right)_{x_{1}}^{\Psi} \mathscr{J}_{\kappa}^{\tau}\left(v f_{2}\right)\left(x_{2}\right) \mid \\
\leq & \frac{1}{\kappa^{2} \Gamma_{\kappa}^{2}(\tau)} \mid \int_{x_{1}}^{x_{2}} \int_{x_{1}}^{x_{2}}\left(\Psi\left(x_{2}\right)-\Psi(\xi)\right)^{\frac{\tau}{\kappa}-1}\left(\Psi\left(x_{2}\right)-\Psi(\varrho)\right)^{\frac{\tau}{\kappa}-1} \Psi^{\prime}(\xi) \mu(\xi) \Psi^{\prime}(\varrho) v(\varrho) \\
& \times\left[\left(f_{1}(\xi)-f_{1}(\varrho)\right)\left(f_{2}(\xi)-f_{2}(\varrho)\right)\right] d \xi d \varrho \mid
\end{aligned}
$$




$$
\begin{aligned}
\leq & \frac{1}{\kappa^{2} \Gamma_{\kappa}^{2}(\tau)} \int_{x_{1}}^{x_{2}} \int_{x_{1}}^{x_{2}}\left(\Psi\left(x_{2}\right)-\Psi(\xi)\right)^{\frac{\tau}{\kappa}-1}\left(\Psi\left(x_{2}\right)-\Psi(\varrho)\right)^{\frac{\tau}{\kappa}-1} \Psi^{\prime}(\xi) \mu(\xi) \Psi^{\prime}(\varrho) \nu(\varrho) \\
& \times\left|\frac{\left(f_{1}(\xi)-f_{1}(\varrho)\right)}{\xi-\varrho}\right|\left|(\xi-\varrho)\left(f_{2}(\xi)-f_{2}(\varrho)\right)\right| d \xi d \varrho \\
\leq & \frac{\left\|f_{1}^{\prime}\right\|_{\infty}}{\kappa^{2} \Gamma_{\kappa}^{2}(\tau)} \int_{x_{1}}^{x_{2}} \int_{x_{1}}^{\vartheta}\left(\Psi\left(x_{2}\right)-\Psi(\xi)\right)^{\frac{\tau}{\kappa}-1}\left(\Psi\left(x_{2}\right)-\Psi(\varrho)\right)^{\frac{\tau}{\kappa}-1} \Psi^{\prime}(\xi) \mu(\xi) \Psi^{\prime}(\varrho) v(\varrho) \\
& \times(\xi-\varrho)\left(\int_{x_{1}}^{x_{2}} f_{2}^{\prime}(\vartheta) d \vartheta\right) d \xi d \varrho \\
\leq & \frac{\left\|f_{1}^{\prime}\right\|_{\infty}}{\kappa^{2} \Gamma_{\kappa}^{2}(\tau)}\left[\int_{x_{1}}^{x_{2}}\left(\Psi\left(x_{2}\right)-\Psi(\xi)\right)^{\frac{\tau}{\kappa}-1} \xi \Psi^{\prime}(\xi) \mu(\xi) d \xi\right. \\
& \times \int_{x_{1}}^{\vartheta}\left(\Psi\left(x_{2}\right)-\Psi(\varrho)\right)^{\frac{\tau}{\kappa}-1} \Psi^{\prime}(\varrho) v(\varrho) d \varrho-\int_{x_{1}}^{x_{2}}\left(\Psi\left(x_{2}\right)-\Psi(\xi)\right)^{\frac{\tau}{\kappa}-1} \Psi^{\prime}(\xi) \mu(\xi) d \xi \\
& \left.\times \int_{x_{1}}^{\vartheta} \varrho\left(\Psi\left(x_{2}\right)-\Psi(\varrho)\right)^{\frac{\tau}{\kappa}-1} \Psi^{\prime}(\varrho) v(\varrho) d \varrho\right] \int_{x_{1}}^{x_{2}} f_{2}^{\prime}(\vartheta) d \vartheta .
\end{aligned}
$$

Hence taking (9) into account, we complete the proof of (17).

Corollary 3.7 Let $\Psi$ be a measurable increasing positive function on $\left(x_{1}, x_{2}\right)$ with continuous derivative $\Psi^{\prime}(\varrho)$ on $\left[x_{1}, x_{2}\right]$. Let $f_{1}:\left[x_{1}, x_{2}\right] \rightarrow \mathbb{R}$ be an absolutely continuous function with $\left(f_{1}^{\prime}\right) \in L^{\infty}\left[x_{1}, x_{2}\right]$, and let $f_{2}:\left[x_{1}, x_{2}\right] \rightarrow \mathbb{R}$ be a nondecreasing function. Suppose that $v:\left[x_{1}, x_{2}\right] \rightarrow \mathbb{R}^{+}$is positive integrable. Then for all $\tau, \kappa>0$, we have

$$
\begin{aligned}
& \mid \frac{\left(\Psi\left(x_{2}\right)-\Psi\left(x_{1}\right)\right)^{\frac{\tau}{\kappa}}}{\Gamma_{\kappa}(\tau+\kappa)}{ }_{x_{1}} \mathscr{I}_{\kappa}^{\tau}\left(v f_{1} f_{2}\right)\left(x_{2}\right)+{ }_{x_{1}}^{\Psi} \mathscr{I}_{\kappa}^{\tau} v\left(x_{2}\right)_{x_{1}}^{\Psi} \mathscr{I}_{\kappa}^{\tau}\left(f_{1} f_{2}\right)\left(x_{2}\right) \\
& \quad-{ }_{x_{1}}^{\Psi} \mathscr{I}_{\kappa}^{\tau}\left(v f_{1}\right)\left(x_{2}\right)_{x_{1}}^{\Psi} \mathscr{I}_{\kappa}^{\tau}\left(f_{2}\right)\left(x_{2}\right)-{ }_{x_{1}}^{\Psi} \mathscr{I}_{\kappa}^{\tau}\left(f_{1}\right)\left(x_{2}\right)_{x_{1}}^{\Psi} \mathscr{I}_{\kappa}^{\tau}\left(v f_{2}\right)\left(x_{2}\right) \mid \\
& \leq \frac{\left\|f_{1}^{\prime}\right\|_{\infty}}{\kappa \Gamma_{\kappa}(\tau)}\left[{ }_{x_{1}}^{\Psi} \mathscr{I}_{\kappa}^{\tau} x_{2} \int_{x_{1}}^{\vartheta}\left(\Psi\left(x_{2}\right)-\Psi(\varrho)\right)^{\frac{\tau}{\kappa}-1} \Psi^{\prime}(\varrho) v(\varrho) d \varrho\right. \\
& \left.\quad-\frac{\left(\Psi\left(x_{2}\right)-\Psi\left(x_{1}\right)\right)^{\frac{\tau}{\kappa}}}{\Gamma_{\kappa}(\tau+\kappa)} \int_{x_{1}}^{\vartheta} \varrho\left(\Psi\left(x_{2}\right)-\Psi(\varrho)\right)^{\frac{\tau}{\kappa}-1} \Psi^{\prime}(\varrho) v(\varrho) d \varrho\right] \int_{x_{1}}^{x_{2}} f_{2}^{\prime}(\vartheta) d \vartheta .
\end{aligned}
$$

Proof Applying Theorem 3.3 with $\mu(\vartheta)=1, \vartheta \in\left[x_{1}, x_{2}\right]$, we obtain the desired result.

Corollary 3.8 Let $\Psi$ be a measurable increasing positive function on $\left(x_{1}, x_{2}\right)$ with continuous derivative $\Psi^{\prime}(\varrho)$ on $\left[x_{1}, x_{2}\right]$. Let $f_{1}:\left[x_{1}, x_{2}\right] \rightarrow \mathbb{R}$ be an absolutely continuous function with $\left(f_{1}^{\prime}\right) \in L^{\infty}\left[x_{1}, x_{2}\right]$, and let $f_{2}:\left[x_{1}, x_{2}\right] \rightarrow \mathbb{R}$ be nondecreasing. Suppose that $\mu:\left[x_{1}, x_{2}\right] \rightarrow \mathbb{R}^{+}$is positive integrable. Then for all $\tau, \kappa>0$, we have

$$
\begin{aligned}
\mid{ }_{x_{1}}^{\Psi} \mathscr{I}_{\kappa}^{\tau} \mu\left(x_{2}\right)_{x_{1}}^{\Psi} \mathscr{I}_{\kappa}^{\tau}\left(v f_{1} f_{2}\right)\left(x_{2}\right)+\frac{\left(\Psi\left(x_{2}\right)-\Psi\left(x_{1}\right)\right)^{\frac{\tau}{\kappa}}}{\Gamma_{\kappa}(\tau+\kappa)}{ }_{x_{1}} \mathscr{I}_{\kappa}^{\tau}\left(\mu f_{1} f_{2}\right)\left(x_{2}\right) \\
\quad-{ }_{x_{1}}^{\Psi} \mathscr{I}_{\kappa}^{\tau}\left(f_{1}\right)\left(x_{2}\right)_{x_{1}}^{\Psi} \mathscr{I}_{\kappa}^{\tau}\left(\mu f_{2}\right)\left(x_{2}\right)-{ }_{x_{1}}^{\Psi} \mathscr{I}_{\kappa}^{\tau}\left(\mu f_{1}\right)\left(x_{2}\right)_{x_{1}}^{\Psi} \mathscr{I}_{\kappa}^{\tau}\left(f_{2}\right)\left(x_{2}\right) \mid \\
\leq \frac{\left\|f_{1}^{\prime}\right\|_{\infty}}{\kappa \Gamma_{\kappa}(\tau)}\left[{ }_{x_{1}}^{\Psi} \mathscr{I}_{\kappa}^{\tau} x_{2} \mu\left(x_{2}\right) \int_{x_{1}}^{\vartheta}\left(\Psi\left(x_{2}\right)-\Psi(\varrho)\right)^{\frac{\tau}{\kappa}-1} \Psi^{\prime}(\varrho) d \varrho\right. \\
\left.\quad-{ }_{x_{1}}^{\Psi} \mathscr{I}_{\kappa}^{\tau} \mu\left(x_{2}\right) \int_{x_{1}}^{\vartheta} \varrho\left(\Psi\left(x_{2}\right)-\Psi(\varrho)\right)^{\frac{\tau}{\kappa}-1} \Psi^{\prime}(\varrho) d \varrho\right] \int_{x_{1}}^{x_{2}} f_{2}^{\prime}(\vartheta) d \vartheta .
\end{aligned}
$$


Proof Applying Theorem 3.3 with $v(\vartheta)=1, \vartheta \in\left[x_{1}, x_{2}\right]$, we obtain the desired result.

Corollary 3.9 Let $\Psi$ be a measurable increasing positive function on $\left(x_{1}, x_{2}\right)$ with continuous derivative $\Psi^{\prime}(\varrho)$ on $\left[x_{1}, x_{2}\right]$. Let $f_{1}:\left[x_{1}, x_{2}\right] \rightarrow \mathbb{R}$ be an absolutely continuous function with $\left(f_{1}^{\prime}\right) \in L^{\infty}\left[x_{1}, x_{2}\right]$, and let $f_{2}:\left[x_{1}, x_{2}\right] \rightarrow \mathbb{R}$ be nondecreasing. Then for all $\tau, \kappa>0$, we have

$$
\begin{aligned}
& \left|\frac{\left(\Psi\left(x_{2}\right)-\Psi\left(x_{1}\right)\right)^{\frac{\tau}{\kappa}}}{\Gamma_{\kappa}(\tau+\kappa)}{ }_{x_{1}} \mathscr{I}_{\kappa}^{\tau}\left(v f_{1} f_{2}\right)\left(x_{2}\right)-{ }_{x_{1}}^{\Psi} \mathscr{I}_{\kappa}^{\tau}\left(f_{1}\right)\left(x_{2}\right)_{x_{1}}^{\Psi} \mathscr{I}_{\kappa}^{\tau}\left(f_{2}\right)\left(x_{2}\right)\right| \\
& \leq \frac{\left\|f_{1}^{\prime}\right\|_{\infty}\left[\frac { \Psi } { 2 \kappa \Gamma _ { \kappa } ( \tau ) } \left[\mathscr{I}_{\kappa}^{\tau} x_{2} \int_{x_{1}}^{\vartheta}\left(\Psi\left(x_{2}\right)-\Psi(\varrho)\right)^{\frac{\tau}{\kappa}-1} \Psi^{\prime}(\varrho) v(\varrho) d \varrho\right.\right.}{\left.\quad-\frac{\left(\Psi\left(x_{2}\right)-\Psi\left(x_{1}\right)\right)^{\frac{\tau}{\kappa}}}{\Gamma_{\kappa}(\tau+\kappa)} \int_{x_{1}}^{\vartheta} \varrho\left(\Psi\left(x_{2}\right)-\Psi(\varrho)\right)^{\frac{\tau}{\kappa}-1} \Psi^{\prime}(\varrho) v(\varrho) d \varrho\right] \int_{x_{1}}^{x_{2}} f_{2}^{\prime}(\vartheta) d \vartheta .}
\end{aligned}
$$

Proof Applying Theorem 3.3 with $\mu(\vartheta)=v(\vartheta)=1, \vartheta \in\left[x_{1}, x_{2}\right]$, we obtain the desired result.

Theorem 3.4 Let $\Psi$ be a measurable increasing positive function on $\left(x_{1}, x_{2}\right)$ with continuous derivative $\Psi^{\prime}(\varrho)$ on $\left[x_{1}, x_{2}\right]$. Let $f_{1}, f_{2}:\left[x_{1}, x_{2}\right] \rightarrow \mathbb{R}$ be absolutely continuous functions, and let $f_{2}:\left[x_{1}, x_{2}\right] \rightarrow \mathbb{R}$ be nondecreasing. Suppose that $\mu, v:\left[x_{1}, x_{2}\right] \rightarrow \mathbb{R}^{+}$are positive integrable. If $f_{1}^{\prime}, f_{2}^{\prime} \in L^{\infty}\left[x_{1}, x_{2}\right]$, then for all $\tau, \kappa>0$, we have

$$
\begin{aligned}
\left.\right|_{x_{1}} ^{\Psi} \mathscr{I}_{\kappa}^{\tau} \mu\left(x_{2}\right)_{x_{1}}^{\Psi} \mathscr{J}_{\kappa}^{\tau}\left(v f_{1} f_{2}\right)\left(x_{2}\right)+{ }_{x_{1}}^{\Psi} \mathscr{J}_{\kappa}^{\tau} v\left(x_{2}\right)_{x_{1}}^{\Psi} \mathscr{I}_{\kappa}^{\tau}\left(\mu f_{1} f_{2}\right)\left(x_{2}\right) \\
\quad-{ }_{x_{1}}^{\Psi} \mathscr{I}_{\kappa}^{\tau}\left(v f_{1}\right)\left(x_{2}\right)_{x_{1}}^{\Psi} \mathscr{I}_{\kappa}^{\tau}\left(\mu f_{2}\right)\left(x_{2}\right)-{ }_{x_{1}}^{\Psi} \mathscr{J}_{\kappa}^{\tau}\left(\mu f_{1}\right)\left(x_{2}\right)_{x_{1}}^{\Psi} \mathscr{I}_{\kappa}^{\tau}\left(v f_{2}\right)\left(x_{2}\right) \mid \\
\leq \frac{\left\|f_{1}^{\prime}\right\|_{\infty}\left\|f_{2}^{\prime}\right\|_{\infty}}{\kappa \Gamma_{\kappa}(\tau)}\left[{ }_{x_{1}}^{\Psi} \mathscr{I}_{\kappa}^{\tau} x_{2}^{2} \mu\left(x_{2}\right) \int_{x_{1}}^{\vartheta}\left(\Psi\left(x_{2}\right)-\Psi(\varrho)\right)^{\frac{\tau}{\kappa}-1} \Psi^{\prime}(\varrho) v(\varrho) d \varrho\right. \\
\quad-2_{x_{1}}^{\Psi} \mathscr{I}_{\kappa}^{\tau} x_{2} \mu\left(x_{2}\right) \int_{x_{1}}^{\vartheta} \varrho\left(\Psi\left(x_{2}\right)-\Psi(\varrho)\right)^{\frac{\tau}{\kappa}-1} \Psi^{\prime}(\varrho) v(\varrho) d \varrho \\
\left.\quad+{ }_{x_{1}}^{\Psi} \mathscr{I}_{\kappa}^{\tau} \mu\left(x_{2}\right) \int_{x_{1}}^{\vartheta} \varrho^{2}\left(\Psi\left(x_{2}\right)-\Psi(\varrho)\right)^{\frac{\tau}{\kappa}-1} \Psi^{\prime}(\varrho) v(\varrho) d \varrho\right] .
\end{aligned}
$$

Proof Considering the left-hand side of (18), we have

$$
\begin{aligned}
& \mid{ }_{x_{1}}^{\Psi} \mathscr{I}_{\kappa}^{\tau} \mu\left(x_{2}\right)_{x_{1}}^{\Psi} \mathscr{I}_{\kappa}^{\tau}\left(v f_{1} f_{2}\right)\left(x_{2}\right)+{ }_{x_{1}}^{\Psi} \mathscr{I}_{\kappa}^{\tau} v\left(x_{2}\right)_{x_{1}}^{\Psi} \mathscr{I}_{\kappa}^{\tau}\left(\mu f_{1} f_{2}\right)\left(x_{2}\right) \\
& \quad-{ }_{x_{1}}^{\Psi} \mathscr{I}_{\kappa}^{\tau}\left(v f_{1}\right)\left(x_{2}\right)_{x_{1}}^{\Psi} \mathscr{I}_{\kappa}^{\tau}\left(\mu f_{2}\right)\left(x_{2}\right)-{ }_{x_{1}}^{\Psi} \mathscr{I}_{\kappa}^{\tau}\left(\mu f_{1}\right)\left(x_{2}\right)_{x_{1}}^{\Psi} \mathscr{J}_{\kappa}^{\tau}\left(v f_{2}\right)\left(x_{2}\right) \mid \\
& \leq \frac{1}{\kappa^{2} \Gamma_{\kappa}^{2}(\tau)} \mid \int_{x_{1}}^{x_{2}} \int_{x_{1}}^{x_{2}}\left(\Psi\left(x_{2}\right)-\Psi(\xi)\right)^{\frac{\tau}{\kappa}-1}\left(\Psi\left(x_{2}\right)-\Psi(\varrho)\right)^{\frac{\tau}{\kappa}-1} \Psi^{\prime}(\xi) \mu(\xi) \Psi^{\prime}(\varrho) v(\varrho) \\
& \times\left[\left(f_{1}(\xi)-f_{1}(\varrho)\right)\left(f_{2}(\xi)-f_{2}(\varrho)\right)\right] d \xi d \varrho \mid \\
& \leq \frac{1}{\kappa^{2} \Gamma_{\kappa}^{2}(\tau)} \int_{x_{1}}^{x_{2}} \int_{x_{1}}^{x_{2}}\left(\Psi\left(x_{2}\right)-\Psi(\xi)\right)^{\frac{\tau}{\kappa}-1}\left(\Psi\left(x_{2}\right)-\Psi(\varrho)\right)^{\frac{\tau}{\kappa}-1} \Psi^{\prime}(\xi) \mu(\xi) \Psi^{\prime}(\varrho) v(\varrho) \\
& \times\left|\frac{\left(f_{1}(\xi)-f_{1}(\varrho)\right)}{\xi-\varrho}\right| \frac{\left(f_{2}(\xi)-f_{2}(\varrho)\right)}{\xi-\varrho} \mid(\xi-\varrho)^{2} d \xi d \varrho \\
& \leq \frac{\left\|f_{1}^{\prime}\right\|_{\infty}\left\|f_{2}^{\prime}\right\|_{\infty}}{\kappa^{2} \Gamma_{\kappa}^{2}(\tau)} \int_{x_{1}}^{x_{2}} \int_{x_{1}}^{\vartheta}\left(\Psi\left(x_{2}\right)-\Psi(\xi)\right)^{\frac{\tau}{\kappa}-1}\left(\Psi\left(x_{2}\right)-\Psi(\varrho)\right)^{\frac{\tau}{\kappa}-1} \Psi^{\prime}(\xi) \mu(\xi) \Psi^{\prime}(\varrho) \nu(\varrho)
\end{aligned}
$$




$$
\begin{aligned}
& \times\left(\xi^{2}-2 \xi \varrho+\varrho^{2}\right) d \xi d \varrho \\
\leq & \frac{\left\|f_{1}^{\prime}\right\|_{\infty}\left\|f_{2}^{\prime}\right\|_{\infty}}{\kappa^{2} \Gamma_{\kappa}^{2}(\tau)}\left[\int_{x_{1}}^{x_{2}}\left(\Psi\left(x_{2}\right)-\Psi(\xi)\right)^{\frac{\tau}{\kappa}-1} \xi^{2} \Psi^{\prime}(\xi) \mu(\xi) d \xi\right. \\
& \times \int_{x_{1}}^{\vartheta}\left(\Psi\left(x_{2}\right)-\Psi(\varrho)\right)^{\frac{\tau}{\kappa}-1} \Psi^{\prime}(\varrho) \nu(\varrho) d \varrho \\
& -2 \int_{x_{1}}^{x_{2}}\left(\Psi\left(x_{2}\right)-\Psi(\xi)\right)^{\frac{\tau}{\kappa}-1} \xi \Psi^{\prime}(\xi) \mu(\xi) d \xi \int_{x_{1}}^{\vartheta} \varrho\left(\Psi\left(x_{2}\right)-\Psi(\varrho)\right)^{\frac{\tau}{\kappa}-1} \Psi^{\prime}(\varrho) v(\varrho) d \varrho \\
& \left.+\int_{x_{1}}^{x_{2}}\left(\Psi\left(x_{2}\right)-\Psi(\xi)\right)^{\frac{\tau}{\kappa}-1} \Psi^{\prime}(\xi) \mu(\xi) d \xi \int_{x_{1}}^{\vartheta} \varrho^{2}\left(\Psi\left(x_{2}\right)-\Psi(\varrho)\right)^{\frac{\tau}{\kappa}-1} \Psi^{\prime}(\varrho) \nu(\varrho) d \varrho\right] .
\end{aligned}
$$

Hence by (9) we complete the proof.

Corollary 3.10 Let $\Psi$ be a measurable increasing positive function on $\left(x_{1}, x_{2}\right)$ with continuous derivative $\Psi^{\prime}(\varrho)$ on $\left[x_{1}, x_{2}\right]$. Let $f_{1}, f_{2}:\left[x_{1}, x_{2}\right] \rightarrow \mathbb{R}$ be absolutely continuous functions, and let $f_{2}:\left[x_{1}, x_{2}\right] \rightarrow \mathbb{R}$ be nondecreasing. Suppose that $v:\left[x_{1}, x_{2}\right] \rightarrow \mathbb{R}^{+}$is positive integrable. If $f_{1}^{\prime}, f_{2}^{\prime} \in L^{\infty}\left[x_{1}, x_{2}\right]$, then for all $\tau, \kappa>0$, we have

$$
\begin{aligned}
& \mid \frac{\left(\Psi\left(x_{2}\right)-\Psi\left(x_{1}\right)\right)^{\frac{\tau}{\kappa}}}{\Gamma_{\kappa}(\tau+\kappa)}{ }_{x_{1}} \mathscr{I}_{\kappa}^{\tau}\left(v f_{1} f_{2}\right)\left(x_{2}\right)+{ }_{x_{1}}^{\Psi} \mathscr{I}_{\kappa}^{\tau} v\left(x_{2}\right)_{x_{1}}^{\Psi} \mathscr{I}_{\kappa}^{\tau}\left(f_{1} f_{2}\right)\left(x_{2}\right) \\
& \quad-{ }_{x_{1}}^{\Psi} \mathscr{I}_{\kappa}^{\tau}\left(v f_{1}\right)\left(x_{2}\right)_{x_{1}}^{\Psi} \mathscr{I}_{\kappa}^{\tau}\left(f_{2}\right)\left(x_{2}\right)-{ }_{x_{1}}^{\Psi} \mathscr{I}_{\kappa}^{\tau}\left(f_{1}\right)\left(x_{2}\right)_{x_{1}}^{\Psi} \mathscr{I}_{\kappa}^{\tau}\left(v f_{2}\right)\left(x_{2}\right) \mid \\
& \leq \frac{\left\|f_{1}^{\prime}\right\|_{\infty}\left\|f_{2}^{\prime}\right\|_{\infty}}{\kappa \Gamma_{\kappa}(\tau)}\left[{ }_{x_{1}}^{\Psi} \mathscr{I}_{\kappa}^{\tau} x_{2}^{2} \int_{x_{1}}^{\vartheta}\left(\Psi\left(x_{2}\right)-\Psi(\varrho)\right)^{\frac{\tau}{\kappa}-1} \Psi^{\prime}(\varrho) v(\varrho) d \varrho\right. \\
& \quad-2_{x_{1}}^{\Psi} \mathscr{I}_{\kappa}^{\tau} x_{2} \int_{x_{1}}^{\vartheta} \varrho\left(\Psi\left(x_{2}\right)-\Psi(\varrho)\right)^{\frac{\tau}{\kappa}-1} \Psi^{\prime}(\varrho) v(\varrho) d \varrho \\
& \left.\quad+\frac{\left(\Psi\left(x_{2}\right)-\Psi\left(x_{1}\right)\right)^{\frac{\tau}{\kappa}}}{\Gamma_{\kappa}(\tau+\kappa)} \int_{x_{1}}^{\vartheta} \varrho^{2}\left(\Psi\left(x_{2}\right)-\Psi(\varrho)\right)^{\frac{\tau}{\kappa}-1} \Psi^{\prime}(\varrho) v(\varrho) d \varrho\right] .
\end{aligned}
$$

Proof Setting $\mu(\vartheta)=1, \vartheta \in\left[x_{1}, x_{2}\right]$, in Theorem 3.4, we obtain the desired result.

Corollary 3.11 Let $\Psi$ be a measurable increasing positive function on $\left(x_{1}, x_{2}\right)$ with continuous derivative $\Psi^{\prime}(\varrho)$ on $\left[x_{1}, x_{2}\right]$. Let $f_{1}, f_{2}:\left[x_{1}, x_{2}\right] \rightarrow \mathbb{R}$ be absolutely continuous functions, and let $f_{2}:\left[x_{1}, x_{2}\right] \rightarrow \mathbb{R}$ be nondecreasing. Suppose that $\mu:\left[x_{1}, x_{2}\right] \rightarrow \mathbb{R}^{+}$is positive integrable. If $f_{1}^{\prime}, f_{2}^{\prime} \in L^{\infty}\left[x_{1}, x_{2}\right]$, then for all $\tau, \kappa>0$, we have

$$
\begin{aligned}
\mid{ }_{x_{1}}^{\Psi} \mathscr{I}_{\kappa}^{\tau} \mu\left(x_{2}\right)_{x_{1}}^{\Psi} \mathscr{I}_{\kappa}^{\tau}\left(f_{1} f_{2}\right)\left(x_{2}\right)+\frac{\left(\Psi\left(x_{2}\right)-\Psi\left(x_{1}\right)\right)^{\frac{\tau}{\kappa}}}{\Gamma_{\kappa}(\tau+\kappa)}{ }_{x_{1}} \mathscr{I}_{\kappa}^{\tau}\left(\mu f_{1} f_{2}\right)\left(x_{2}\right) \\
\quad-{ }_{x_{1}}^{\Psi} \mathscr{I}_{\kappa}^{\tau}\left(f_{1}\right)\left(x_{2}\right)_{x_{1}}^{\Psi} \mathscr{I}_{\kappa}^{\tau}\left(\mu f_{2}\right)\left(x_{2}\right)-{ }_{x_{1}}^{\Psi} \mathscr{I}_{\kappa}^{\tau}\left(\mu f_{1}\right)\left(x_{2}\right)_{x_{1}}^{\Psi} \mathscr{I}_{\kappa}^{\tau}\left(f_{2}\right)\left(x_{2}\right) \mid \\
\leq \frac{\left\|f_{1}^{\prime}\right\|_{\infty}\left\|f_{2}^{\prime}\right\|_{\infty}}{\kappa \Gamma_{\kappa}(\tau)}\left[{ }_{x_{1}}^{\Psi} \mathscr{I}_{\kappa}^{\tau} x_{2}^{2} \mu\left(x_{2}\right) \int_{x_{1}}^{\vartheta}\left(\Psi\left(x_{2}\right)-\Psi(\varrho)\right)^{\frac{\tau}{\kappa}-1} \Psi^{\prime}(\varrho) d \varrho\right. \\
\quad-2_{x_{1}}^{\Psi} \mathscr{I}_{\kappa}^{\tau} x_{2} \mu\left(x_{2}\right) \int_{x_{1}}^{\vartheta} \varrho\left(\Psi\left(x_{2}\right)-\Psi(\varrho)\right)^{\frac{\tau}{\kappa}-1} \Psi^{\prime}(\varrho) d \varrho \\
\left.+{ }_{x_{1}}^{\Psi} \mathscr{I}_{\kappa}^{\tau} \mu\left(x_{2}\right) \int_{x_{1}}^{\vartheta} \varrho^{2}\left(\Psi\left(x_{2}\right)-\Psi(\varrho)\right)^{\frac{\tau}{\kappa}-1} \Psi^{\prime}(\varrho) d \varrho\right] .
\end{aligned}
$$


Proof Setting $v(\vartheta)=1, \vartheta \in\left[x_{1}, x_{2}\right]$, in Theorem 3.4, we obtain the desired result.

Corollary 3.12 Let $\Psi$ be a measurable increasing positive function on $\left(x_{1}, x_{2}\right)$ with continuous derivative $\Psi^{\prime}(\varrho)$ on $\left[x_{1}, x_{2}\right]$. Let $f_{1}, f_{2}:\left[x_{1}, x_{2}\right] \rightarrow \mathbb{R}$ be absolutely continuous functions, and let $f_{2}:\left[x_{1}, x_{2}\right] \rightarrow \mathbb{R}$ be nondecreasing. If $f_{1}^{\prime}, f_{2}^{\prime} \in L^{\infty}\left[x_{1}, x_{2}\right]$, then for all $\tau, \kappa>0$, we have

$$
\begin{aligned}
& \left|\frac{\left(\Psi\left(x_{2}\right)-\Psi\left(x_{1}\right)\right)^{\frac{\tau}{\kappa}}}{\Gamma_{\kappa}(\tau+\kappa)}{ }_{x_{1}} \mathscr{I}_{\kappa}^{\tau}\left(f_{1} f_{2}\right)\left(x_{2}\right)-{ }_{x_{1}}^{\Psi} \mathscr{I}_{\kappa}^{\tau}\left(f_{1}\right)\left(x_{2}\right)_{x_{1}}^{\Psi} \mathscr{I}_{\kappa}^{\tau}\left(f_{2}\right)\left(x_{2}\right)\right| \\
& \leq \frac{\left\|f_{1}^{\prime}\right\|_{\infty}\left\|f_{2}^{\prime}\right\|_{\infty}}{2 \kappa \Gamma_{\kappa}(\tau)}\left[{ }_{x_{1}}^{\Psi} \mathscr{I}_{\kappa}^{\tau} x_{2}^{2} \int_{x_{1}}^{\vartheta}\left(\Psi\left(x_{2}\right)-\Psi(\varrho)\right)^{\frac{\tau}{\kappa}-1} \Psi^{\prime}(\varrho) d \varrho\right. \\
& \quad-2_{x_{1}}^{\Psi} \mathscr{I}_{\kappa}^{\tau} x_{2} \int_{x_{1}}^{\vartheta} \varrho\left(\Psi\left(x_{2}\right)-\Psi(\varrho)\right)^{\frac{\tau}{\kappa}-1} \Psi^{\prime}(\varrho) d \varrho \\
& \left.\quad+{ }_{x_{1}}^{\Psi} \mathscr{I}_{\kappa}^{\tau} \mu\left(x_{2}\right) \int_{x_{1}}^{\vartheta} \varrho^{2}\left(\Psi\left(x_{2}\right)-\Psi(\varrho)\right)^{\frac{\tau}{\kappa}-1} \Psi^{\prime}(\varrho) d \varrho\right] .
\end{aligned}
$$

Proof Setting $\mu(\vartheta)=v(\vartheta)=1, \vartheta \in\left[x_{1}, x_{2}\right]$, in Theorem 3.4, we obtain the desired result.

\section{Particular cases}

Here we present some inequalities in terms of the Riemann-Liouville $\kappa$-fractional integrals, which are the particular cases of the main results.

Theorem 4.1 Suppose that $\Phi:\left[x_{1}, x_{2}\right] \rightarrow \mathbb{R}$ is absolutely continuous on $\left[x_{1}, x_{2}\right]$ with $\left(\Phi^{\prime}\right)^{2} \in L_{1}\left[x_{1}, x_{2}\right]$ and that $\mu, v:\left[x_{1}, x_{2}\right] \rightarrow \mathbb{R}^{+}$are positive integrable. Then for all $\tau, \kappa>0$, we have

$$
\begin{aligned}
& {\left[x_{1} \mathscr{I}_{\kappa}^{\tau} \mu\left(x_{2}\right)\right]\left[x_{1} \mathscr{I}_{\kappa}^{\tau}\left(v \Phi^{2}\right)\left(x_{2}\right)\right]+\left[x_{1} \mathscr{I}_{\kappa}^{\tau} v\left(x_{2}\right)\right]\left[x_{1} \mathscr{I}_{\kappa}^{\tau}\left(\mu \Phi^{2}\right)\left(x_{2}\right)\right] } \\
& \quad-2\left[x_{1} \mathscr{I}_{\kappa}^{\tau}(\mu \Phi)\left(x_{2}\right)\right]\left[x_{1} \mathscr{I}_{\kappa}^{\tau}(v \Phi)\left(x_{2}\right)\right] \\
& \leq \frac{1}{\kappa \Gamma_{\kappa}(\tau)} \int_{x_{1}}^{x_{2}}\left[{ }_{x_{1}} \mathscr{I}_{\kappa}^{\tau} x_{2} \mu\left(x_{2}\right) \int_{x_{1}}^{\vartheta}\left(x_{2}-\varrho\right)^{\frac{\tau}{\kappa}-1} v(\varrho) d \varrho\right. \\
&\left.-{ }_{x_{1}} \mathscr{I}_{\kappa}^{\tau} \mu\left(x_{2}\right) \int_{x_{1}}^{\vartheta} \varrho\left(x_{2}-\varrho\right)^{\frac{\tau}{\kappa}-1} v(\varrho) d \varrho\right]\left(\Phi^{\prime}(\vartheta)\right)^{2} d \vartheta
\end{aligned}
$$

Proof Applying Theorem 3.1 with $\Psi(\vartheta)=\vartheta$ gives the proof of the theorem.

Theorem 4.2 Suppose that $f_{1}, f_{2}:\left[x_{1}, x_{2}\right] \rightarrow \mathbb{R}$ are absolutely continuous functions with $\left(f_{1}^{\prime}\right)^{2} \in L_{1}\left[x_{1}, x_{2}\right]$ and $\left(f_{2}^{\prime}\right)^{2} \in L_{1}\left[x_{1}, x_{2}\right]$ and that $\mu, v:\left[x_{1}, x_{2}\right] \rightarrow \mathbb{R}^{+}$are positive integrable. Then for all $\tau, \kappa>0$, we have

$$
\begin{aligned}
\mid x_{1} \mathscr{I}_{\kappa}^{\tau} \mu\left(x_{2}\right)_{x_{1}} \mathscr{I}_{\kappa}^{\tau}\left(v f_{1} f_{2}\right)\left(x_{2}\right)+{ }_{x_{1}} \mathscr{I}_{\kappa}^{\tau} v\left(x_{2}\right)_{x_{1}} \mathscr{I}_{\kappa}^{\tau}\left(\mu f_{1} f_{2}\right)\left(x_{2}\right) & \\
& -{ }_{x_{1}} \mathscr{I}_{\kappa}^{\tau}\left(v f_{1}\right)\left(x_{2}\right)_{x_{1}} \mathscr{I}_{\kappa}^{\tau}\left(\mu f_{2}\right)\left(x_{2}\right)-{ }_{x_{1}} \mathscr{I}_{\kappa}^{\tau}\left(\mu f_{1}\right)\left(x_{2}\right)_{x_{1}} \mathscr{I}_{\kappa}^{\tau}\left(v f_{2}\right)\left(x_{2}\right) \mid \\
\leq & \frac{1}{\kappa \Gamma_{\kappa}(\tau)}\left(\int _ { x _ { 1 } } ^ { x _ { 2 } } \left[{ }_{x_{1}} \mathscr{I}_{\kappa}^{\tau} x_{2} \mu\left(x_{2}\right) \int_{x_{1}}^{\vartheta}\left(x_{2}-\varrho\right)^{\frac{\tau}{\kappa}-1} v(\varrho) d \varrho\right.\right. \\
& \left.\left.\quad-{ }_{x_{1}} \mathscr{I}_{\kappa}^{\tau} \mu\left(x_{2}\right) \int_{x_{1}}^{\vartheta} \varrho\left(x_{2}-\varrho\right)^{\frac{\tau}{\kappa}-1} v(\varrho) d \varrho\right]\left(f_{1}^{\prime}(\vartheta)\right)^{2} d \vartheta\right)^{\frac{1}{2}}
\end{aligned}
$$




$$
\begin{aligned}
& \times\left(\int _ { x _ { 1 } } ^ { x _ { 2 } } \left[{ }_{x_{1}} \mathscr{I}_{\kappa}^{\tau} x_{2} \mu\left(x_{2}\right) \int_{x_{1}}^{\vartheta}\left(x_{2}-\varrho\right)^{\frac{\tau}{\kappa}-1} v(\varrho) d \varrho\right.\right. \\
& \left.\left.-{ }_{x_{1}} \mathscr{I}_{\kappa}^{\tau} \mu\left(x_{2}\right) \int_{x_{1}}^{\vartheta} \varrho\left(x_{2}-\varrho\right)^{\frac{\tau}{\kappa}-1} v(\varrho) d \varrho\right]\left(f_{2}^{\prime}(\vartheta)\right)^{2} d \vartheta\right)^{\frac{1}{2}} .
\end{aligned}
$$

Proof Applying Theorem 3.2 with $\Psi(\vartheta)=\vartheta$ gives the proof of the theorem.

Similarly, we can get several new inequalities in terms of the Riemann-Liouville $\kappa$ fractional integrals for $\Psi(\vartheta)=\vartheta$ in Theorems 3.3-3.4. Also, employing Corollaries 3.13.12 for $\Psi(\vartheta)=\vartheta$ results in various new inequalities.

Remark 4.1 We can also establish other types of new inequalities by taking the following assumptions:

i. Setting $\mu(\vartheta)=v(\vartheta)$ and $\Psi(\vartheta)=\vartheta$ throughout the paper.

ii. Setting $\mu(\vartheta)=v(\vartheta)=1$ and $\Psi(\vartheta)=\vartheta$ throughout the paper.

Remark 4.2 If we take $\kappa=1$, then all established results reduce to the work of Bezziou et al. [5].

Remark 4.3 Setting $\mu(\vartheta)=v(\vartheta), \kappa=1$, and $\Psi(\vartheta)=\vartheta$ in Theorems 3.1-3.4 restores the results of Bezziou et al. [4].

\section{Concluding remarks}

In this present paper, we derived some double-weighted generalized fractional integral inequalities by employing the generalized Riemann-Liouville $\kappa$-fractional integrals containing another function $\Psi$ in the kernels, where $\Psi$ is integrable, measurable, positive, and monotone. We can quickly form many new fractional integral inequalities for different fractional definitions by considering Remark 2.2.

Acknowledgements

Not applicable.

Funding

The author T. Abdeljawad would like to thank Prince Sultan University for funding this work through research group Nonlinear Analysis Methods in Applied Mathematics (NAMAM) group number RG-DES-2017-01-17.

Availability of data and materials

None.

Competing interests

The authors declare that they have no competing interests.

Authors' contributions

All authors contributed equally, and they read and approved the final manuscript for publication.

\section{Author details}

'Department of Mathematics, Shaheed Benazir Bhutto University, Sheringal, Upper Dir, Khyber Pakhtoonkhwa, Pakistan. ${ }^{2}$ Department of Mathematics, College of Arts and Sciences, Prince Sattam bin Abdulaziz University, Wadi Aldawaser 11991, Saudi Arabia. ${ }^{3}$ Department of Engineering Science, Kermanshah University of Technology, Kermanshah, Iran. ${ }^{4}$ Department of Mathematics, Faculty of Engineering and Natural Sciences, Bahçeşehir University, 34349 Istanbul, Turkey. ${ }^{5}$ Department of Mathematics and General Sciences, Prince Sultan University, P.O. Box 66833, Riyadh, 11586, Kingdom of Saudi Arabia. ${ }^{6}$ Department of Medical Research, China Medical University, Taichung 40402, Taiwan. ${ }^{7}$ Department of Computer Science and Information Engineering, Asia University, Taichung, 40402, Taiwan. 


\section{Publisher's Note}

Springer Nature remains neutral with regard to jurisdictional claims in published maps and institutional affiliations.

\section{Received: 13 April 2020 Accepted: 13 July 2020 Published online: 18 July 2020}

\section{References}

1. Alshabanat, A., Jeli, M., Kumar, S., Samet, B.: Generalization of Caputo-Fabrizio fractional derivative and applications to electrical circuits. Front. Phys. 8, 64 (2020)

2. Awan, K.M., Pecaric, J., Rehman, A.: Steffensen's generalization of Chebyshev inequality. J. Math. Inequal. 9(1), 155-163 (2015)

3. Belarbi, S., Dahmani, Z:: On some new fractional integral inequalities. J. Inequal. Pure Appl. Math. 10(3), 1-12 (2009)

4. Bezziou, M., Dahmani, Z., Khameli, A.: Some weighted inequalities of Chebyshev type via RL-approach. Mathematica 60(83), 12-20 (2018)

5. Bezziou, M., Dahmani, Z., Khameli, A.: On some double-weighted fractional integral inequalities. Sarajevo J. Math. 15(28), 23-36 (2019)

6. Cerone, P., Dragomir, S.S.: A refinement of the Gruss inequality and applications. Tamkang J. Math. 38(1), 37-49 (2007)

7. Chebyshev, P.L.: Sur les expressions approximatives des intégrales définies par les autres prises entre les mêmes limites. Proc. Math. Soc. Charkov. 2, 93-98 (1882)

8. Dahmani, Z:: New inequalities in fractional integrals. Int. J. Nonlinear Sci. 9, 493-497 (2010)

9. Dahmani, Z.: New inequalities in fractional integrals. Int. J. Nonlinear Sci. 9(4), 493-497 (2010)

10. Dahmani, Z: The Riemann-Liouville operator to generate some new inequalities. Int. J. Nonlinear Sci. 12, 452-455 (2011)

11. Dahmani, Z: About some integral inequalities using Riemann-Liouville integrals. Gen. Math. 20(4), 63-69 (2012)

12. Dahmani, Z., Benzidane, A.: New inequalities using Q-fractional theory. Bull. Math. Anal. Appl. 4(1), 190-196 (2012)

13. Dahmani, Z., Bounoua, M.D.: Further results on Chebyshev and Steffensen inequalities. Kyungpook Math. J. 58, 55-66 (2018)

14. Dahmani, Z., Tabharit, L.: On weighted Gruss type inequalities via fractional integration. J. Adv. Res. Pure Math. 2, 31-38 (2010)

15. Diaz, R., Pariglan, E.: On hypergeometric functions and Pochhammer k-symbol. Divulg. Mat. 15(2), 179-192 (2007)

16. Dragomir, S.S.: A generalization of Gruss's inequality in inner product spaces and applications. J. Math. Anal. Appl. 237(1), 74-82 (1999)

17. Ghanbari, B., Kumar, S., Kumar, R.: A study of behaviour for immune and tumor cells in immunogenetic tumour model with non-singular fractional derivative. Chaos Solitons Fractals 133, 109619 (2020)

18. Goufo, E.F., Kumar, S., Mugisha, S.B.: Similarities in a fifth-order evolution equation with and with no singular kernel. Chaos Solitons Fractals 130, 109467 (2020)

19. Habib, S., Mubeen, S., Naeem, M.N.: Chebyshev type integral inequalities for generalized $k$-fractional conformable integrals. J. Inequal. Spec. Funct. 9(4), 53-65 (2018)

20. Huang, C.J., Rahman, G., Nisar, K.S., Ghaffar, A., Qi, F.: Some inequalities of Hermite-Hadamard type for k-fractional conformable integrals. Aust. J. Math. Anal. Appl. 16(1), 1-9 (2019)

21. Iqbal, S., Mubeen, S., Tomar, M.: On Hadamard k-fractional integrals. J. Fract. Calc. Appl. 9, 255-267 (2018)

22. Jarad, F., Abdeljawad, T., Alzabut, J.: Generalized fractional derivatives generated by a class of local proportional derivatives. Eur. Phys. J. 226, 3457-3471 (2017). https://doi.org/10.1140/epjst/e2018-00021-7

23. Jarad, F., Ugurlu, E., Abdeljawad, T., Baleanu, D.: On a new class of fractional operators. Adv. Differ. Equ. 2017, 247 (2017)

24. Katugampola, U.N.: Approach to a generalized fractional integral. Appl. Math. Comput. 218, 860-865 (2011)

25. Khan, T.U., Khan, M.A.: Generalized conformable fractional integral operators. J. Comput. Appl. Math. 346, 378-389 (2018). https://doi.org/10.1016/j.cam.2018.07.018

26. Kilbas, A.A., Srivastava, H.M., Trujillo, J.J.: Theory and Application of Fractional Differential Equations. Elsevier, Amsterdam (2006)

27. Kuang, J.C.: Applied Inequalities. Shandong Sciences and Technologie Press, Jinan (2004)

28. Kumar, S.: A new fractional modeling arising in engineering sciences and its analytical approximate solution. Alex. Eng. J. 52(4), 813-819 (2013)

29. Kumar, S., Ahmadian, A., Kumar, R., Kumar, D., Singh, J., Baleanu, D., Salimi, M.: An efficient numerical method for fractional SIR epidemic model of infectious disease by using Bernstein wavelets. Mathematics 8(4), 558 (2020)

30. Kumar, S., Ghosh, S., Samet, B., Goufo, E.F.: An analysis for heat equations arises in diffusion process using new Yang-Abdel-Aty-Cattani fractional operator. Math. Methods Appl. Sci. 43, 6062-6080 (2020)

31. Kumar, S., Kumar, R., Agarwal, R.P., Samet, B.: A study of fractional Lotka-Volterra population model using Haar wavelet and Adams-Bashforth-Moulton methods. Math. Methods Appl. Sci. 43(8), 5564-5578 (2020)

32. Kumar, S., Nisar, K.S., Kumar, R., Cattani, C., Samet, B.: A new Rabotnov fractional-exponential function based fractional derivative for diffusion equation under external force. Math. Methods Appl. Sci. 43(7), 4460-4471 (2020). https://doi.org/10.1002/mma.6208

33. Kwun, Y.C., Farid, G., Nazeer, W., Ullah, S., Kang, S.M.: Generalized Riemann-Liouville $k$-fractional integrals associated with Ostrowski type inequalities and error bounds of Hadamard inequalities. IEEE Access 6, 64946-64953 (2018)

34. McD Mercer, A.: An improvement of the Gruss inequality. J. Inequal. Pure Appl. Math. 10(4), Art. 93 (2005)

35. Mitrinovic, D.S.: Analytic Inequalities. Springer, Berlin (1970)

36. Mitrinovic, D.S., Pecaric, J.E., Fink, A.M.: Classical and New Inequalities in Analysis. Kluwer Academic, Dordrecht (1993)

37. Mubeen, S., Habibullah, G.M.: k-Fractional integrals and application. Int. J. Contemp. Math. Sci. 7, 89-94 (2012)

38. Nisar, K.S., Qi, F., Rahman, G., Mubeen, S., Arshad, M.: Some inequalities involving the extended gamma function and the Kummer confluent hypergeometric $k$-function. J. Inequal. Appl. 2018, 135 (2018)

39. Nisar, K.S., Rahman, G., Choi, J., Mubeen, S., Arshad, M.: Certain Gronwall type inequalities associated with Riemann-Liouville k- and Hadamard k-fractional derivatives and their applications. East Asian Math. J. 34(3), 249-263 (2018) 
40. Nisar, K.S., Rahman, G., Khan, A.: Some new inequalities for generalized fractional conformable integral operators. Adv Differ. Equ. 2019, 427 (2019). https://doi.org/10.1186/s13662-019-2362-3

41. Nisar, K.S., Rahman, G., Khan, A., Tassaddiq, A., Abouzaid, M.S.: Certain generalized fractional integral inequalities. AIMS Math. 5(2), 1588-1602 (2020). https://doi.org/10.3934/math.2020108

42. Nisar, K.S., Rahman, G., Mehrez, K.: Chebyshev type inequalities via generalized fractional conformable integrals. J. Inequal. Appl. 2019, 245 (2019). https://doi.org/10.1186/s13660-019-2197-1

43. Nisar, K.S., Tassadiq, A., Rahman, G., Khan, A.: Some inequalities via fractional conformable integral operators. J. Inequal. Appl. 2019, 217 (2019). https://doi.org/10.1186/s13660-019-2170-z

44. Ostrowski, A.M.: On an integral inequality. Aequ. Math. 4, 358-373 (1970)

45. Qi, F., Rahman, G., Hussain, S.M., Du, W.S., Nisar, K.S.: Some inequalities of Čebyšev type for conformable $k$-fractional integral operators. Symmetry 10, 614 (2018). https://doi.org/10.3390/sym10110614

46. Rahman, G., Abdeljawad, T., Jarad, F., Khan, A., Nisar, K.S.: Certain inequalities via generalized proportional Hadamard fractional integral operators. Adv. Differ. Equ. 2019, 454 (2019). https://doi.org/10.1186/s13662-019-2381-0

47. Rahman, G., Abdeljawad, T., Jarad, F., Nisar, K.S.: Bounds of generalized proportional fractional integrals in general form via convex functions and their applications. Mathematics 8,113 (2020). https://doi.org/10.3390/math8010113

48. Rahman, G., Abdeljawad, T., Jarad, F., Nisar, K.S.: Bounds of generalized proportional fractional integrals in general form via convex functions and their applications. Mathematics 8,113 (2020). https://doi.org/10.3390/math8010113

49. Rahman, G., Abdeljawad, T., Khan, A., Nisar, K.S.: Some fractional proportional integral inequalities. J. Inequal. Appl. 2019, 244 (2019). https://doi.org/10.1186/s13660-019-2199-z

50. Rahman, G., Khan, A., Abdeljawad, T., Nisar, K.S.: The Minkowski inequalities via generalized proportional fractional integral operators. Adv. Differ. Equ. 2019, 287 (2019). https://doi.org/10.1186/s13662-019-2229-7

51. Rahman, G., Nisar, K.S., Abdeljawad, T.: Certain Hadamard proportional fractional integral inequalities. Mathematics 8 , 504 (2020). https://doi.org/10.3390/math8040504

52. Rahman, G., Nisar, K.S., Abdeljawad, T., Ullah, S.: Certain fractional proportional integral inequalities via convex functions. Mathematics 8, 222 (2020). https://doi.org/10.3390/math8020222

53. Rahman, G., Nisar, K.S., Abdeljawad, T., Ullah, S.: Certain fractional proportional integral inequalities via convex functions. Mathematics 8, 222 (2020). https://doi.org/10.3390/math8020222

54. Rahman, G., Nisar, K.S., Ghaffar, A., Qi, F.: Some inequalities of the Grüss type for conformable $k$-fractional integral operators. Rev. R. Acad. Cienc. Exactas Fís. Nat., Ser. A Mat. 114, 9 (2020). https://doi.org/10.1007/s13398-019-00731-3

55. Rahman, G., Nisar, K.S., Mubeen, S., Choi, J.: Certain inequalities involving the $(k, \rho)$-fractional integral operator. Far East J. Math. Sci.: FJMS 103(11), 1879-1888 (2018)

56. Rahman, G., Nisar, K.S., Qi, F.: Some new inequalities of the Grüss type for conformable fractional integrals. AIMS Math 3(4), 575-583 (2018)

57. Rahman, G., Ullah, Z., Khan, A., Set, E., Nisar, K.S.: Certain Chebyshev type inequalities involving fractional conformable integral operators. Mathematics 7, 364 (2019). https://doi.org/10.3390/math7040364

58. Samko, S.G., Kilbas, A.A., Marichev, O.I.: Fractional Integrals and Derivatives, Theory and Applications. Gordon \& Breach, Yverdon (1993)

59. Sarikaya, M.Z., Budak, H.: Generalized Ostrowski type inequalities for local fractional integrals. Proc. Am. Math. Soc. 145(4), 1527-1538 (2017)

60. Sarikaya, M.Z., Dahmani, Z., Kiris, M.E., Ahmad, F.: (k, s)-Riemann-Liouville fractional integral and applications. Hacet. J. Math. Stat. 45(1), 77-89 (2016)

61. Set, E., Tomar, M., Sarikaya, M.Z:: On generalized Grüss type inequalities for $k$-fractional integrals. Appl. Math. Comput. 269, 29-34 (2015)

62. Sharma, B., Kumar, S., Cattani, C., Baleanu, D.: Nonlinear dynamics of Cattaneo-Christov heat flux model for third-grade power-law fluid. J. Comput. Nonlinear Dyn. 15(1), 011009 (2020). https://doi.org/10.1115/1.4045406

63. Tassaddiq, A., Rahman, G., Nisar, K.S., Samraiz, M.: Certain fractional conformable inequalities for the weighted and the extended Chebyshev functionals. Adv. Differ. Equ. 2020, 96 (2020). https://doi.org/10.1186/s13662-020-2543-0

64. Veeresha, P., Prakasha, D.G., Kumar, S.: A fractional model for propagation of classical optical solitons by using nonsingular derivative. Math. Methods Appl. Sci., 1-15 (2020). https://doi.org/10.1002/mma.6335

\section{Submit your manuscript to a SpringerOpen ${ }^{\circ}$ journal and benefit from:}

- Convenient online submission

- Rigorous peer review

- Open access: articles freely available online

- High visibility within the field

- Retaining the copyright to your article

Submit your next manuscript at $\gg$ springeropen.com 